\title{
Vocal Frequency Estimation and Voicing State Prediction with Surface EMG PATtern ReCognition
}

by

\author{
Winston De Armas
}

A thesis submitted in conformity with the requirements for the degree of Masters of Health Science

Graduate Department of Institute of Biomaterials and Biomedical Engineering University of Toronto

Copyright (C) 2013 by Winston De Armas 


\begin{abstract}
Vocal Frequency Estimation and Voicing State Prediction with Surface EMG Pattern Recognition

Winston De Armas

Masters of Health Science

Graduate Department of Institute of Biomaterials and Biomedical Engineering University of Toronto
\end{abstract}

2011

Most electrolarynges do not allow hands-free use or pitch modulation. This study presents the potential of pattern recognition to support electrolarynx use by predicting fundamental frequency (F0) and voicing state (VS) from neck surface EMG and respiratory trace. Respiratory trace and neck surface EMG were collected from 10 normal, adult males (18-60 years old) during different vocal tasks. Time-domain features were extracted from both signals, and a Support Vector Machine (SVM) classifier was employed to model F0 and VS. An average mean-squared-error (MSE) of $8.21 \pm 3.5$ semitones $^{2}$ was achieved for the estimation of vocal frequency. An average classification accuracy of $78.05 \pm 6.3 \%$ was achieved for the prediction of voicing state from EMG and $65.24 \pm 7.8 \%$ from respiratory trace. Our results show that pattern classification of neck-muscle EMG and respiratory trace has merit in the prediction of F0 and VS during vocalization. 


\section{Acknowledgements}

All praise and honor is due to Jesus Christ, my guide and strength throughout project. I would not be where I am today without You.

To my mother, father and brother: Thank you so much for all the financial, emotional and spiritual support you have given me that has been my anchor as well as my guide and compass throughout my entire life. Thank you also for all the motivational conversations, endless prayers, hugs and life lessons.

Thank you to Dr. Tom Chau for the invaluable opportunity to conduct research with you. The experiences I have had in this lab have been invigorating and rewarding. Thank you especially for being so thoughtful, patient and understanding on so many occasions through the ups and downs of this thesis. You have helped me more than you can know.

To Khondaker Mamun: thank you for your guidance and assistance with all aspects of my data collection and analysis. I am indebted to you for all the late nights, early mornings and your general thoughtfulness and dedication to the success of my project. I would also like to acknowledge my appreciation to Ka Lun Tam and Pierre Duez who have provided hardware and software support this project in their role as engineers. Their assistance has always been prompt and of the utmost quality.

To my friend Maria: Thank you for your friendship and support throughout this Masters journey. You have been there for me through all the dark times and bright times. When I didn't believe in myself, you always believed in me. Thank you for seeing the strengths in me that I myself cannot.

Thank you to Dr. Jan Andrysek, Dr. Tiago Falk, Dr. Tim Bressman and Loralee Mclean and for the feedback and direction they contributed. 


\section{Table of Contents}

Chapter 1 - Introduction ..................................................................... 1

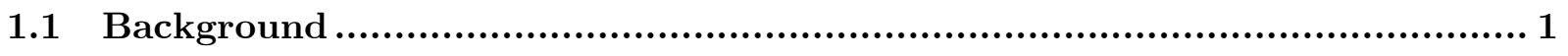

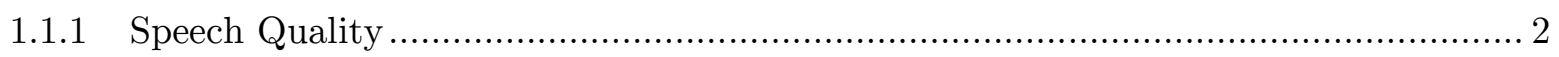

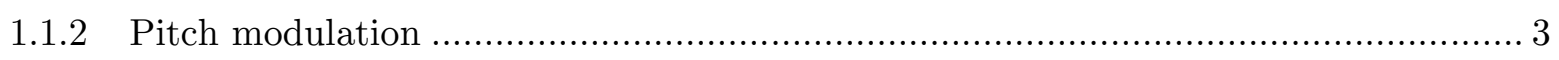

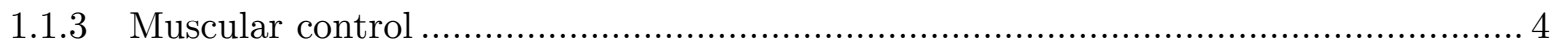

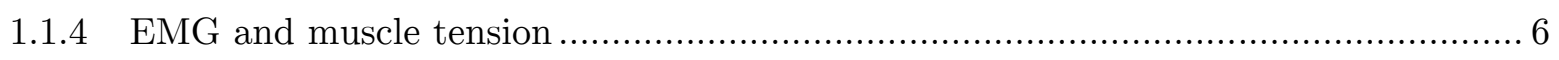

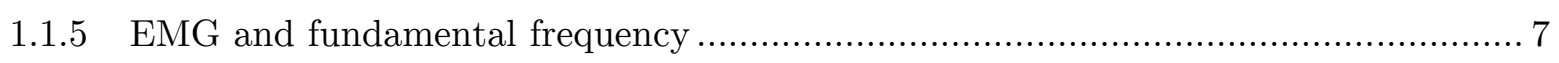

1.1.6 Accurate prediction of F0 from EMG ……….................................................. 8

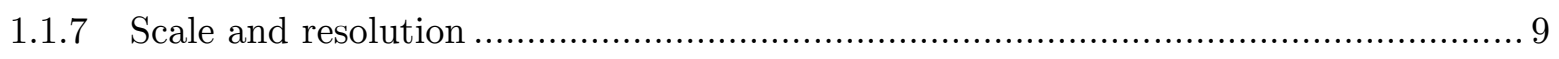

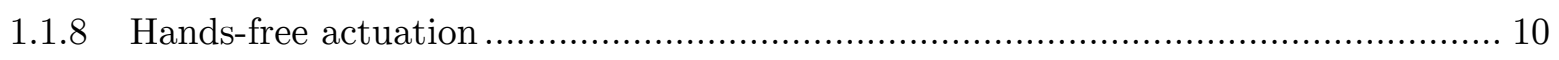

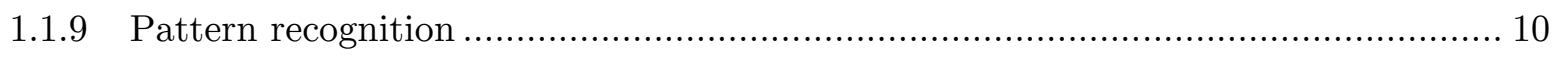

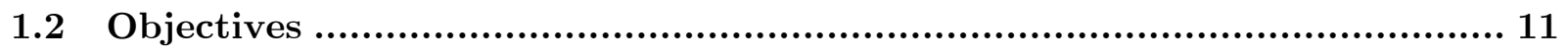

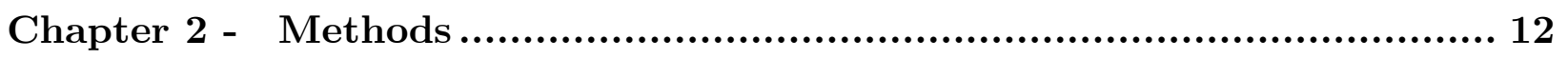

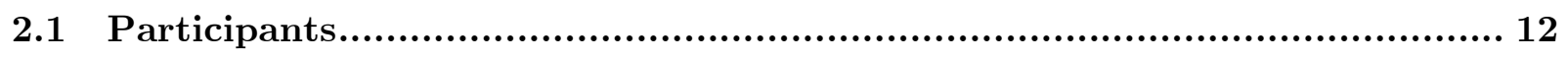

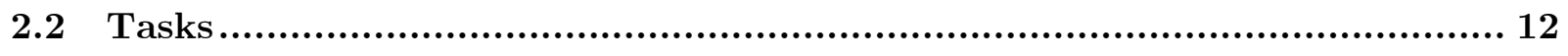

$2.3 \quad$ Experimental protocol.......................................................................... 13

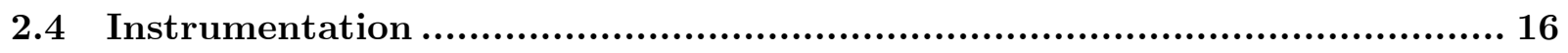

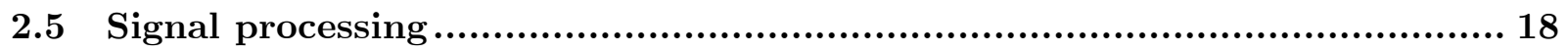

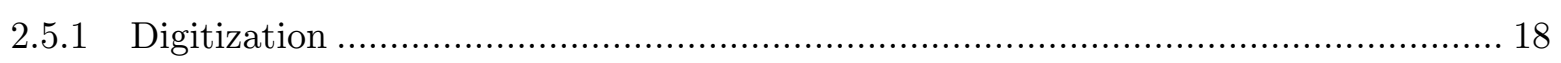

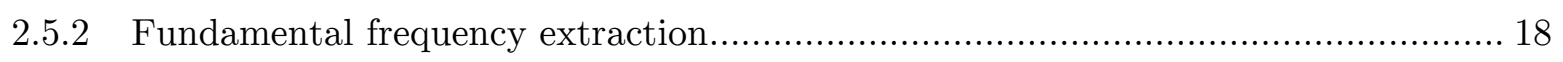

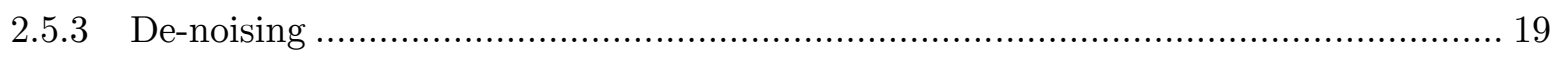

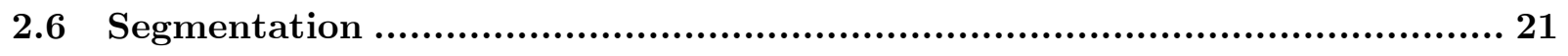

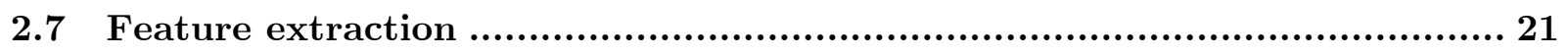




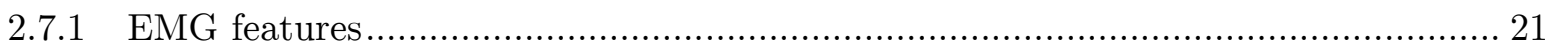

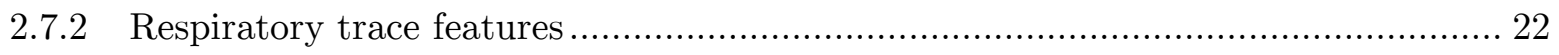

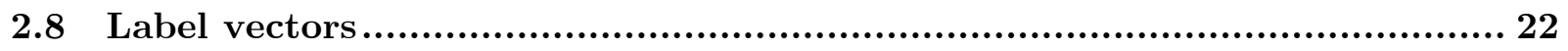

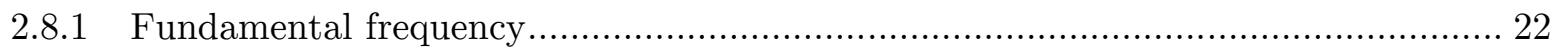

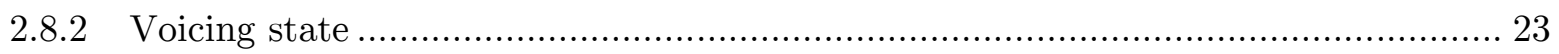

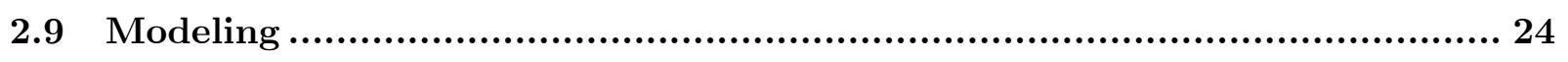

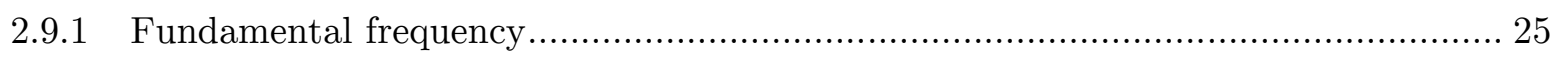

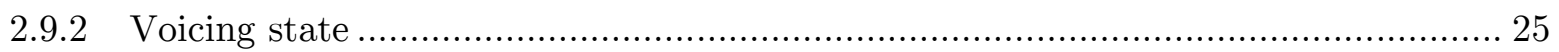

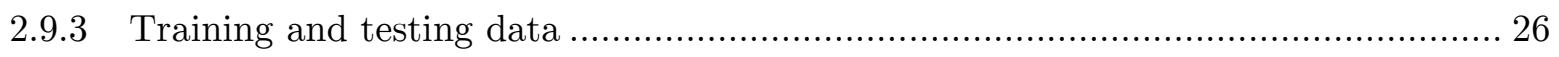

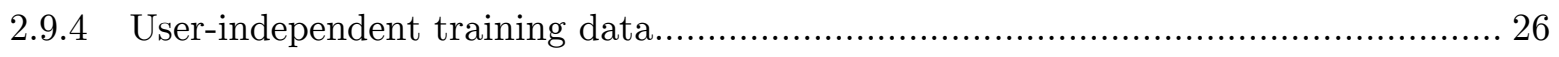

Chapter 3 - Results .................................................................... 28

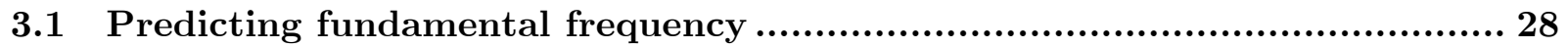

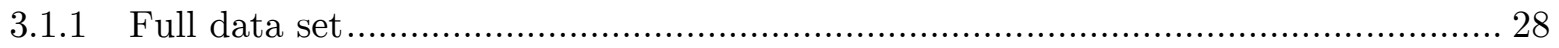

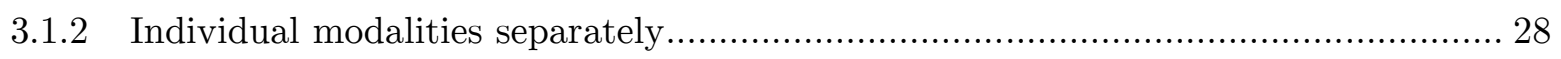

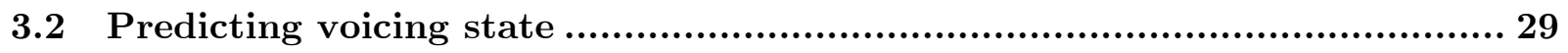

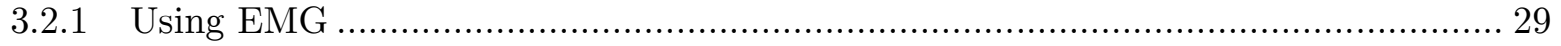

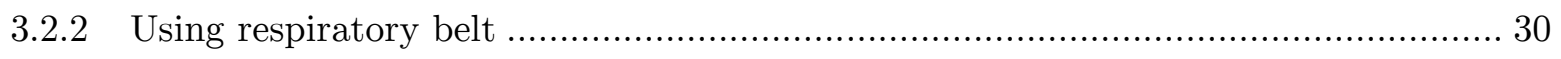

3.3 User Independent Training Data .................................................. 30

Chapter 4 - Discussion ............................................................ 32

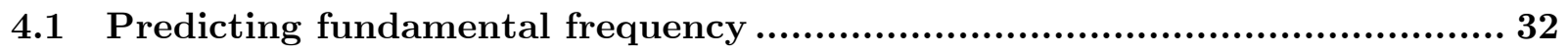

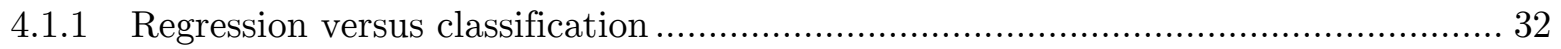

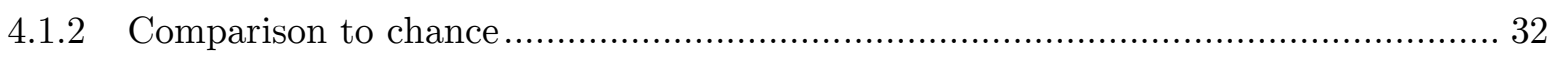

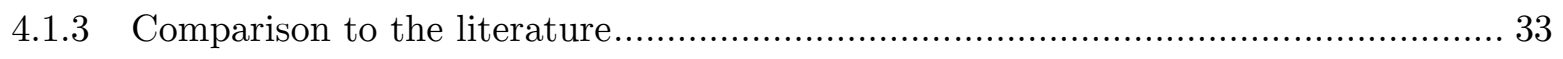

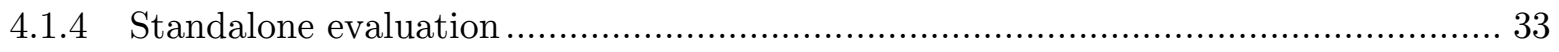

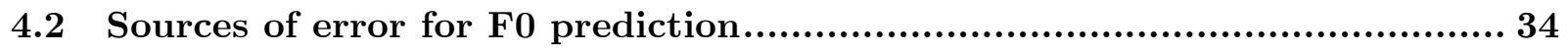

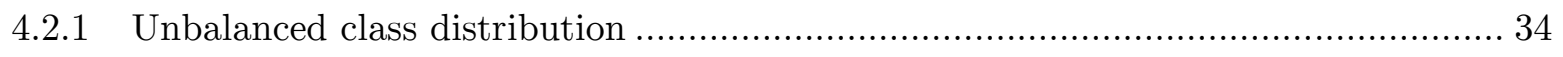

4.2.2 Use of sEMG rather than intramuscular EMG ............................................ 35

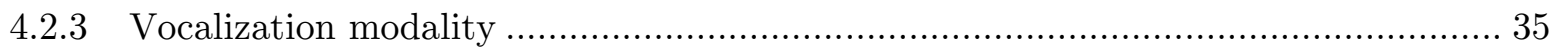




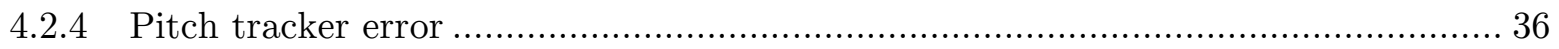

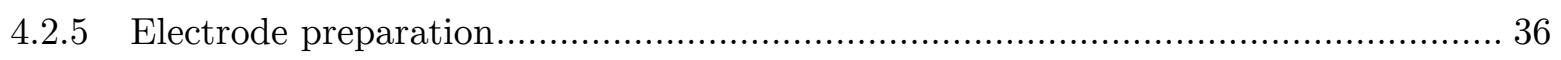

4.3 Predicting voicing state ....................................................... 36

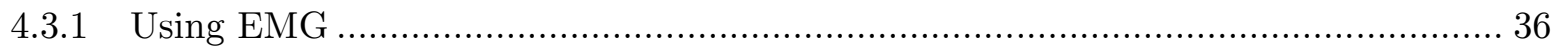

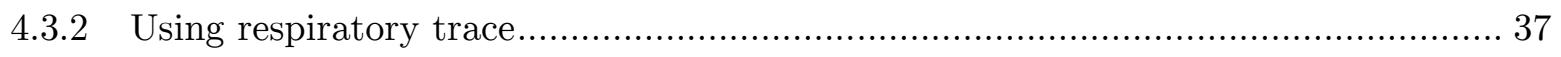

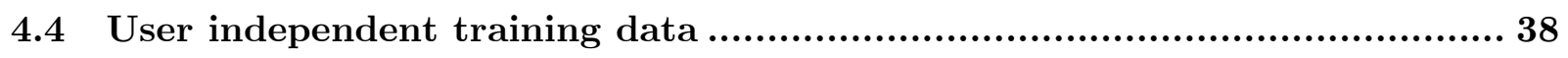

Chapter 5 - Future work ............................................................ 39

5.1 Robustness ....................................................................... 39

5.2 User training for user-independent models ....................................... 39

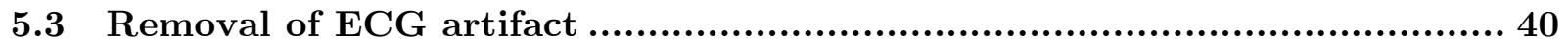

Chapter 6 - Conclusions .............................................................. 41

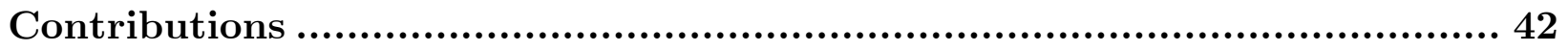

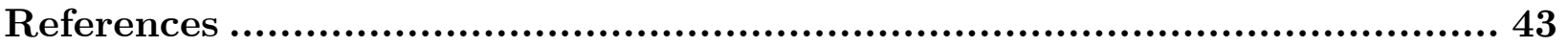




\section{Abbreviations}

\begin{tabular}{|c|c|}
\hline $\mathrm{AR}$ & Autoregressive \\
\hline CF0 & Continuous Fundamental Frequency \\
\hline $\mathrm{CT}$ & Cricothyroid \\
\hline CVS & Change in Voicing State \\
\hline DF0 & Discrete Fundamental Frequency \\
\hline $\mathrm{DWT}$ & Discrete Wavelet Transform \\
\hline ECG & Electrocardiography \\
\hline $\mathrm{EL}$ & Electrolarynx \\
\hline EMG & Electromyography \\
\hline F0 & Fundamental Frequency \\
\hline GMM & Gaussian Mixture Models \\
\hline $\mathrm{IH}$ & Infrahyoid \\
\hline jnd & Just Noticeable Difference \\
\hline MAV & Maximum Amplitude Value \\
\hline MSE & Mean Squared Error \\
\hline $\mathrm{R}^{2}$ & Squared Correlation Coefficient \\
\hline RAPT & Robust Algorithm for Pitch Tracking \\
\hline $\mathrm{RBF}$ & Radial Basis Function \\
\hline RMS & Root Mean Square \\
\hline sEMG & Surface Electromyography \\
\hline $\mathrm{SH}$ & Suprahyoid \\
\hline SNR & Signal to Noise Ratio \\
\hline St. & Semitones \\
\hline STFT & Short Time Fourier Transform \\
\hline
\end{tabular}


SVM Support Vector Machines

VS Voicing State

WL Waveform Length 


\section{List of Tables}

Table 1: Pitch of even tones in semitones ......................................... 13

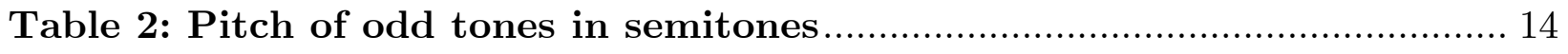

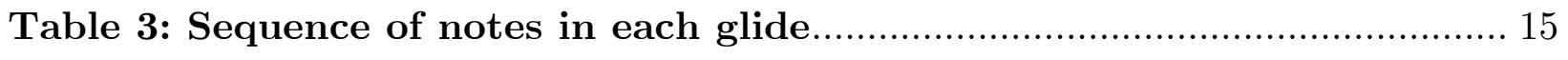

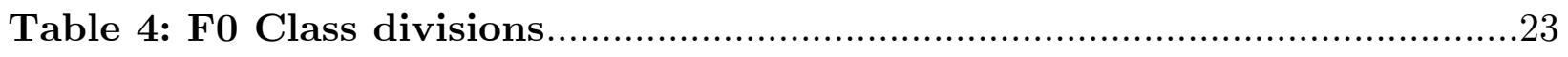

Table 5: CVS values and definitions ................................................. 24 


\section{List of Figures}

Figure 1: Infrahyoid muscle groups in the neck ……................................................ 6

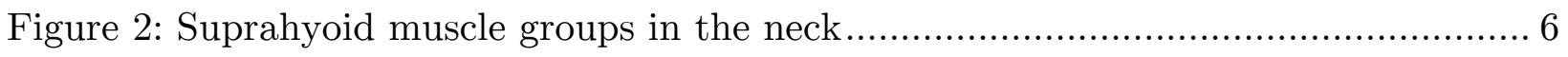

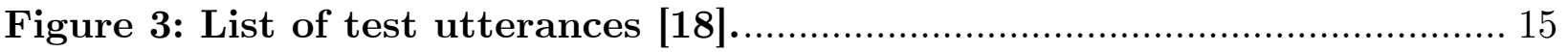

Figure 4: Photograph of electrode positions 1 and 2 on the neck of participant 1 ......... 16

Figure 5: Block diagram depicting flow of data in experimental setup ......................... 17

Figure 6: Infrahyoid EMG before (top) and after (bottom) wavelet de-noising ............. 20

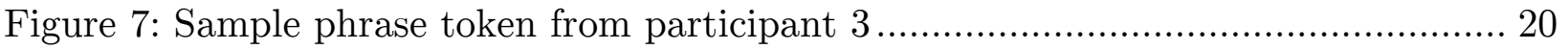

Figure 8: Data analysis configurations for predicting fundamental frequency (F0) ....... 25

Figure 9: Process flow steps outlining the techniques describing the Methods .............. 27

Figure 10: Average mean squared error of classification and regression models for full

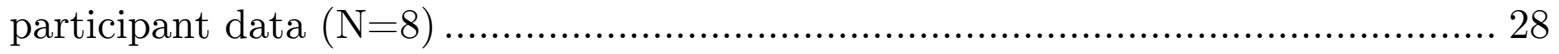

Figure 11: Average mean squared error of models trained on individual modalities

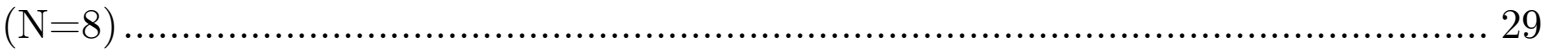

Figure 12: Specificity and sensitivity of voicing state prediction using EMG (N=8) _... 29

Figure 13: Performance of voicing state prediction trained on respiratory trace

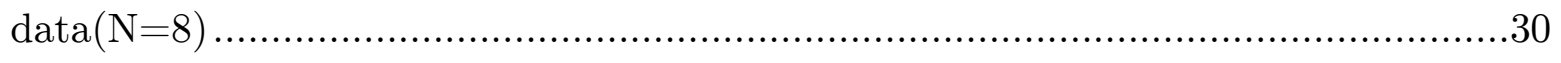

Figure 14: MSE comparison for user-specific and user-independent training data $(\mathrm{N}=8)$ 


\section{Chapter 1 - Introduction}

\subsection{Background}

Patients with malignant tumors in the throat frequently need to have their larynx completely removed after radiotherapy and chemotherapy have failed. The procedure is referred to in the literature as a laryngectomy and it also involves disconnection of the trachea from the pharynx. An opening, or tracheostoma, is permanently incised into the patient's neck and the trachea is rerouted through this aperture. This effectively separates the airway from the nasal, buccal and esophageal passageways. In the absence of the larynx, normal speech becomes impossible because the primary channel of communication is missing. In addition, physical restrictions can prevent some patients from becoming adept at alternative communication channels like esophageal and tracheo-esophageal speech without excessive effort. An external speech prosthesis becomes necessary to restore functional vocalization for these patients [1]. The electrolarynx is far less physically demanding and easier to learn. As a result, the majority of laryngectomees use it as their primary mode of verbal communication [2-4].

The electrolarynx features a transducer that can generate pulses in the range of natural human speech. An oscillator produces an input signal to the windings of an electromagnet, which in turn oscillates against the plastic diaphragm. Actuating the electrolarynx while pressed against the throat sends vibrations through the neck muscles. This, in turn, produces a vibration in the pharynx that duplicates the natural vibration of the vocal cords. The patient can then move and adjust his palate, throat and lips as necessary to shape the sound into intelligible speech. 
Unfortunately, the archetypal electrolarynx suffers from a monotonous tone and the inconvenience of requiring manual control. The former inhibits intelligibility and limits the paralinguistic content of speech, while the latter precludes two-handed tasks during conversation [5]. Variation in fundamental frequency during vocal communication contains a large amount of information about speaker emotion and intention (e.g. question or command; sarcastic or serious) that is important within a social context. In addition, speech with variable intonation has a significant advantage in terms of intelligibility [6] over fixed-frequency intonation. Because the electrolarynx oscillator circuit produces an input signal at a set frequency, the prosodic features of speech tend to be lost.

Meltzner and Hillman [7] showed that, of all attempted acoustic enhancements (reduction of noise and adding low-frequency harmonics), the inclusion of a normal modulation scheme for the fundamental frequency had the largest impact on perceived naturalness of electrolaryngeal speech. Providing an intuitive, hands-free mechanism of controlling initiation, termination and fundamental frequency of the device is an important initial step towards automatic pitch modulation.

\subsubsection{Speech Quality}

The basic concept behind current electrolarynx technology was patented over 40 years ago [8] yet only recently has there has begun to be some focus on improving the inherent deficits in quality, prosody and user interface. In particular, the quality of speech from electrolarynx has received significant attention. To provide some examples, Qi and Weinberg [9] were able to enhance the low-frequency components of the output speech, while Cole et al. [10] have successfully used noise reduction algorithms to 
minimize electrolaryngeal noise. Houston et al. [11] developed a novel electrolarynx that used digital signal processing to produce a better quality output.

\subsubsection{Pitch modulation}

Of more relevance, however, is the research focusing on providing some degree of pitch modulation to the electrolarynx. Current technologies can be classified according to the extent of control that they allow the user over fundamental frequency. Most do not allow any manipulation of pitch (e.g. Western Electric 5A, Amplicode and Nuvois), while some offer a choice between two preset frequencies for a male and for a female respectively (e.g. Servox-inton). The TruTone ${ }^{\mathrm{TM}}$ electrolarynx provides relatively fine control over frequency but reliable hand and finger control are requirements, since pitch is modulated via a finger-controlled force-sensitive sensor on the body of the device. Most users find it difficult and frustrating to control pitch by varying activation pressures using muscles with activation patterns that are not normally voice-related. This feature often results in awkward vocal inflection. Moreover, the transducer location associated with the hands-free mounting system is reportedly sub-optimal for the majority of users (Kubert et al., 2009).

Two strategies predominate the literature:

1. Superimpose a more natural pitch modulation contour onto the speech, without any direct control.

2. Provide the user with hands-free direct control over the fundamental frequency of the electrolaryngeal output using some physiological parameter. 
Most of the research has been focused on the first category. Ma et al. [12] was able to use post-processing to replace the flat electrolaryngeal contour with a normal one. Meltzner and Hillman [7] added a normal frequency contour to recordings of electrolaryngeal speech with positive results. These techniques cannot be implemented in real time, however, since they require pre-recording the speech. Goldstein et al. [13] used the amplitude of the infrahyoid (IH) muscle EMG envelope to provide a frequency contour. Pitch control was achieved in proportion to the level of suprathreshold EMG energy, with greater energy corresponding to higher f0. Participants produced fluctuation in pitch throughout their sentences, yet had difficulty consistently differentiating questions versus statements through intonational contrasts. Saikachi et al. [14] used the amplitude of electrolaryngeal speech to modulate the fundamental frequency. While these may be carried out in real time and results show improved speech quality, the information contained within the prosodic features of speech are not regained and can often be obfuscated $[5,14]$.

The second category is more promising in terms of regaining this information reliably. However, the only significant study thus far has been on the use of exhalation pressure to control pitch. A resistive component is used at the tracheostoma to measure airflow [15], but this proved to be very physically demanding and difficult to control. Only 2 of 16 participants, both of whom were already proficient at esophageal speech, were able to achieve any conscious control over pitch.

\subsubsection{Muscular control}

During vocalization, the intrinsic and extrinsic laryngeal muscles are the groups with overt voice-related activation patterns, and they appear to work antagonistically to control pitch. More specifically, cricothyroid muscle (CT) contraction has been shown to 
increase the fundamental frequency (f0), while infrahyoid muscle contraction lowers it [16]. In addition, the frequency range from $80 \mathrm{~Hz}$ to $300 \mathrm{~Hz}$ appears to be separable into two distinct regions where either CT or infrahyoid muscle activity is the predominant controlling factor [17]. These are referred to in the literature as laryngeal states, each characterized by different laryngeal configurations and controlled by different physiological factors. At higher frequencies, CT is the strongest controlling factor of f0 [18]. In contrast, CT activation drops and activation of the infrahyoid muscles has been shown to increase at very low frequencies. In particular, the sternohyoid $(\mathrm{SH})$ and sternothyroid (ST) muscles show consistently high EMG activation in the range of speech $(80-150 \mathrm{~Hz})$ with an inverse relationship between the level of activation and f0 (Roubeau et al. 1997). This supports the physiological understanding that the extrinsic laryngeal muscles control fundamental frequency by manipulating the vertical position of the larynx, while CT does so by modulating the length of the vocal cords. It should be noted that the quality and quantity of the extrinsic musculature available postlaryngectomy is very case-specific.

Stepp et al. [19] conducted a study that looked at the performance of different electrode positions on the face and neck of laryngectomy patients as EMG control sources for the electrolarynx. They found that the submental muscles and the infrahyoid muscles were the two optimal positions. 


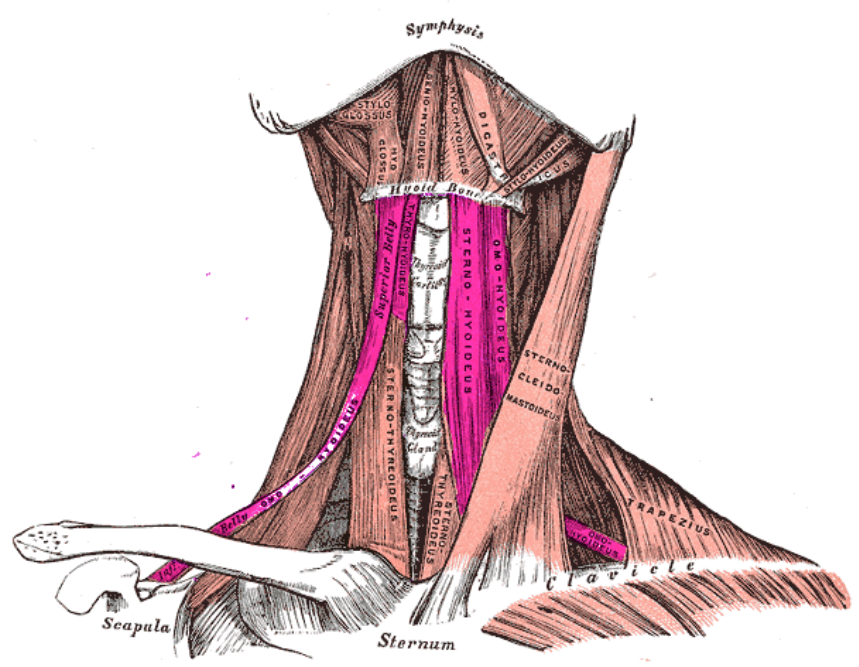

Figure 1: Infrahyoid muscle groups in the neck

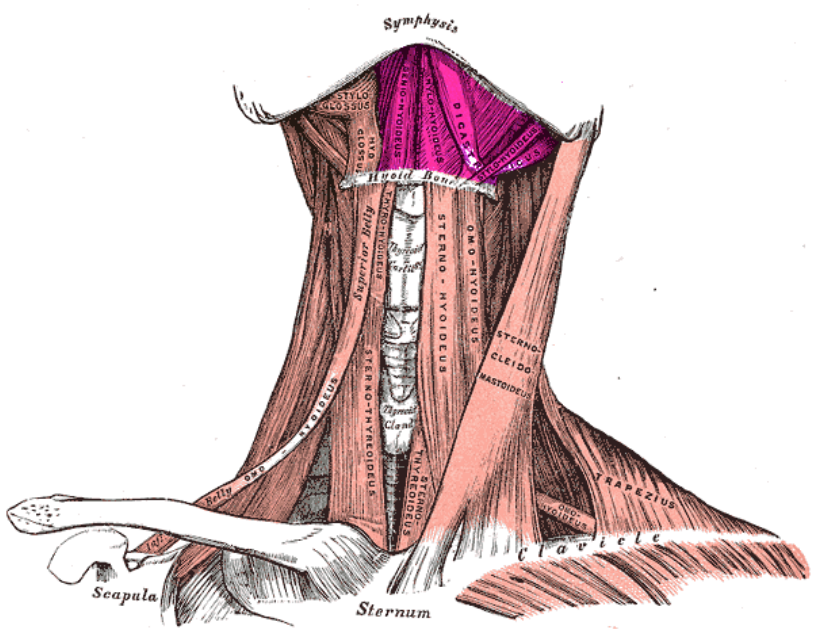

Figure 2: Suprahyoid muscle groups in the neck

\subsubsection{EMG and muscle tension}

EMG has enjoyed considerable success as a control signal in the development of limb prostheses [20-23] and human-computer interfaces [24,25]. In large part, this success can be attributed to the ease and non-invasive nature of acquisition [26], as well as the strong correlation between muscle tension and the amplitude of the associated EMG [27]. 


\subsubsection{EMG and fundamental frequency}

There have been many correlational studies attempting to quantify the influence of CT and infrahyoid activation on fundamental frequency variation. In particular, Shipp et al. [28] found a strong correlation and used regression analysis to extract a linear, predictive relationship between pitch and CT activation for 4 different subjects. As expected, there was significant inter-subject variability. Of course, the pathology implies absence or lack of control over CT muscles, but this approach could be tailored to permit pitch prediction within the $80-150 \mathrm{~Hz}$ range based on infrahyoid activation instead.

The correlation between pitch and infrahyoid activation is not as strong as that between pitch and CT activation, so it is expected that prediction would not be equally accurate [16]. However, the theoretical accuracy of such a predictive relationship is really just a first step. The predictive relationship tries to find a natural first solution to work from, but humans can conceivably develop skill by self-initiated error detection and correction through intrinsic and augmented feedback [29].

Goldstein et al. [13] showed that voluntary control over infrahyoid muscle tension could be achieved by visualizing changes in vocalization frequency in the lower register, thus allowing for indirect modulation of EMG activity. A further study defined a universal, linear positive transform function between EMG activity level and output pitch and explained it to normal participants. After moderate training, participants were able to regulate their infrahyoid muscle activity to consistently produce intonational contrasts that were large enough to distinguish between declarative and interrogative intentions [5]. However, this study focused on infrahyoid control in a broad sense, and participants 
were not encouraged to develop the finer control over pitch regulation that is necessary to provide a wider, normal range of intonation. In addition, while Heaton et al. [30] indicate the existence of a consistent relationship between the intended pitch and EMG amplitude for individuals, there is significant variability across different subjects. This high variability is largely due to inherent physiological differences (e.g. in muscle geometry, ratios of slow- to fast-twitch fibers, recruitment patterns and firing frequencies) and its prevalence suggests that a universal transform function between EMG activity and pitch cannot be defined for all subjects if it is to enable fine regulation of pitch. Control over the infrahyoid muscles appears to be either inherent or easily acquired since normal participants learned to use them even prior to formal training [31], so it is reasonable to imagine that finer control can be developed with training. There is a notable absence of any consideration for variation in the frequency components of infrahyoid muscle EMG with fundamental frequency. Participants in the study conducted by Goldstein et al. [5] were able to elevate infrahyoid muscle activity to increase pitch. This is in contrast to the role of the infrahyoid muscles in normal speech and is evidence of a new pitch regulation mechanism being assimilated.

\subsubsection{Accurate prediction of F0 from EMG}

From the literature, it was speculated that only two studies attempted to accurately predict F0 from correlated physiological signals $[32,28]$. Shipp et al. used logistic regression to uncover a correlation coefficient of $0.93 \pm 0.02$ between normalized intramuscular cricothyroid (CT) EMG and phonation frequency. Even though the result was very promising, the CT muscles are accessible only through invasive needle electrodes. Nakamura et al. used Gaussian Mixture Models (GMM) with surface EMG of different facial muscles but were not able to achieve an EMG-frequency correlation coefficient greater than 0.49 . 
Nonetheless, Nakamura et al. suggested the potential benefit of moving electrode positions to the neck area to capture vocal fold information more accurately. Indeed, the intrinsic and extrinsic laryngeal muscles in the neck have more overt voice-related activation patterns than the facial muscles $[18,16,28]$. The CT muscles control pitch by adjusting the tension in the vocal cords, while the infrahyoid and suprahyoid (SH) muscle groups adjust the vertical position of the hyoid bone and thus the length of the air passage between the base of the skull and the vocal cords. With this in mind, the accessibility of the IH and SH groups by surface EMG makes them better candidates for prediction of F0 than the facial muscles targeted by Nakamura et al.

\subsubsection{Scale and resolution}

Humans perceive pitch intervals logarithmically rather than linearly (that is to say, a

frequency, $f$, is perceived to be equally distant from $f x$ as $f x$ is from $f x^{2}$ where $x$ is a real number). There is a need, therefore to discuss pitch variation in terms of perceptual rather than physical intervals. Expressing pitch in a psycho-acoustic scale helps to preserve equivalence in perception of pitch intervals. The semitone scale has been shown to be most suitable for this type of representation of f0 contours in speech [33].

Humans can aurally distinguish intervals as fine as a quartertone within the $100 \mathrm{~Hz}$ range but the distinction must be noted between this just noticeable difference (jnd) and the smallest information-carrying unit. Research suggests that the semitone interval is the smallest melodic unit that is used to convey meaning in non-tonal languages, with room for minor tuning error. More specifically, pitch differences of as small as 1.5 
semitones can transmit meaning to some degree. Moreover, 2 equivalent intervals would convey the same meaning even if they were off by as much as the jnd [34].

\subsubsection{Hands-free actuation}

Goldstein et al. [13] designed a method of using surface EMG of the infrahyoid muscles to provide on/off control for an electrolarynx. When the EMG signal exceeds a predetermined initiation threshold, the device activates. Similarly, when the signal drops below the termination threshold, the device turns off. Voice initiation was as fast as with normal voice, but voice termination was significantly slower.

Subjects were taught to achieve initiation by visualizing a low-pitch vocalization (thus increasing infrahyoid muscle activation and, in turn, EMG amplitude) and termination by relaxing the muscle group. Voice termination was therefore achieved passively. Since termination is normally an active process involving muscle contraction, EMG activation would be prolonged past the desired termination point, resulting in an undesirable increase in VTT (voice termination time) [5]. In addition, requiring users to learn additional skills augments the cognitive load needed to operate the device. Ideally, user intention should be predicted from natural, already-learned activities. The muscular activation and breathing patterns that occur at the onset and offset of speech carry unique properties and they can be detectable using pattern classification of EMG and respiratory trace signals.

\subsubsection{Pattern recognition}

Surface EMG has achieved considerable success as a control signal in the development of limb prostheses. Machine learning methods have been used both for classifying limb postures as well as for quantifying joint torque [35-38]. The breathing patterns that 
occur at the onset and offset of speech carry unique properties, as do the muscular activation patterns that distinguish vocal and non-vocal activity. These patterns can be detectable using pattern classification of EMG and respiratory trace signals. The success of these methods at quantifying torque from surface EMG can also be extended to the estimation of $\mathrm{F} 0$.

\subsection{Objectives}

The objectives of this study were to:

1. Develop a model capable of estimating the voicing state and fundamental frequency of vocalization in adult males using surface electromyography from the infrahyoid and suprahyoid muscles.

2. Develop a model capable of predicting the voicing state in adult males using the respiratory trace signal. 


\section{Chapter 2 - Methods}

We observed vocal activity in a representative group of participants while collecting EMG and respiratory trace data. We used data this to train the models we described in the Objectives section above.

\subsection{Participants}

Ten adult males with age 18-60 years (mean $29.22 \pm 6.4$ ) and no history of voice or neuromuscular disorders were recruited. Normal participants were recruited to use their outcomes as a proxy for potential performance of laryngectomees with some remaining strap musculature. The primary goal of the study was to validate the experimental procedure and operation of the equipment. The data files of participants 2 and 4 were corrupted and were therefore not included in the study. Males were chosen because their EMG activity in the target muscles is significantly higher than that of females [16]. The study was approved by the research ethics boards of Holland Bloorview Kids Rehabilitation Hospital, and University of Toronto, Toronto, Canada. Participants gave their written informed consent before taking part in the study.

\subsection{Tasks}

In order to observe as wide a variety of vocal activities as possible, data were collected from each participant performing 3 kinds of vocal tasks:

1) tones - discrete, sustained notes with a constant pitch using the vowel /a/ [28]

2) glides - continuous slides from one pitch to another using the vowel /a/ [16] 
3) phrases - standard speech tokens repeated in a normal speaking voice and at a relaxed pace $[18,32]$. These tokens were drawn from the literature and were chosen carefully to include various stress placements [18].

\subsection{Experimental protocol}

For each task, auditory stimuli were electronically generated using custom written MATLAB software to provide a standard for the participant to vocally replicate. Table 1 and Table $\mathbf{2}$ outline the order in which tones were presented. All frequencies are presented in semitones (st.) relative to $90 \mathrm{~Hz}$. Participants were asked to repeat each tone twice and hold it for 8 seconds. Even tones were presented first, followed by odd tones.

Table 1: Pitch of even tones in semitones

\begin{tabular}{|c|c|}
\hline Tone \# & Pitch in st. \\
\hline 1 & 0 \\
\hline 2 & 2 \\
\hline 3 & 4 \\
\hline 4 & 6 \\
\hline$\cdot$ & $\cdot$ \\
\hline$\cdot$ & $\cdot$ \\
\hline$\cdot$ & $\cdot$ \\
\hline 11 & 22 \\
\hline 12 & 24 \\
\hline
\end{tabular}




\section{Table 2: Pitch of odd tones in semitones}

\begin{tabular}{|c|c|}
\hline Tone \# & Pitch in st. \\
\hline 1 & 1 \\
\hline 2 & 3 \\
\hline 3 & 5 \\
\hline 4 & 7 \\
\hline$\cdot$ & $\cdot$ \\
\hline$\cdot$ & $\cdot$ \\
\hline 11 & 21 \\
\hline 12 & 23 \\
\hline
\end{tabular}

Table 3 outlines the order of the glides. Each glide lasted 6s. We used 2 major scales to fully present the 12 notes in each octave. 12 repetitions of each glide were obtained from each participant. Note that the wide frequency range of 90-360 Hz was chosen to be as comprehensive as possible and account for variation in the vocal abilities of participants. 
Table 3: Sequence of notes in each glide

\begin{tabular}{|c|c|c|}
\hline $\begin{array}{c}\text { Glide } \\
\#\end{array}$ & Direction & Sequence of notes in st. \\
\hline 1 & ascending & $0-2-4-5-7-9-11-12$ \\
\hline 2 & ascending & $12-14-16-17-19-21-23-24$ \\
\hline 3 & descending & $24-23-21-19-17-16-14-12$ \\
\hline 4 & descending & $12-11-9-7-5-4-2-0$ \\
\hline 5 & ascending & $1-3-5-6-8-10-12-13$ \\
\hline 6 & ascending & $13-15-17-18-20-22-24-25$ \\
\hline 7 & descending & $25-24-22-20-18-17-15-13$ \\
\hline 8 & descending & $13-12-10-8-6-5-3-1$ \\
\hline
\end{tabular}

Twenty repetitions of each of the utterances shown in Figure $\mathbf{3}$ were obtained from each participant. The underlined words indicate the placement of emphasis in the sentence.

Figure 3: List of test utterances [18].

Bold type indicates sentence stress.

1. Bev loves Bob.

2. Bev loves Bob.

3. Bev loves Bob.

4. Bev loves Bob.

5. Bev loves Bob?

6. Bev loves Bob?

7. Bev loves Bob?

8. Bev loves Bob?

9. He had plans to leave. (Blueprints to drop off.)

10. He had plans to leave. (To depart.)

11 . He had plans to leave.

12. He had plans to leave. 


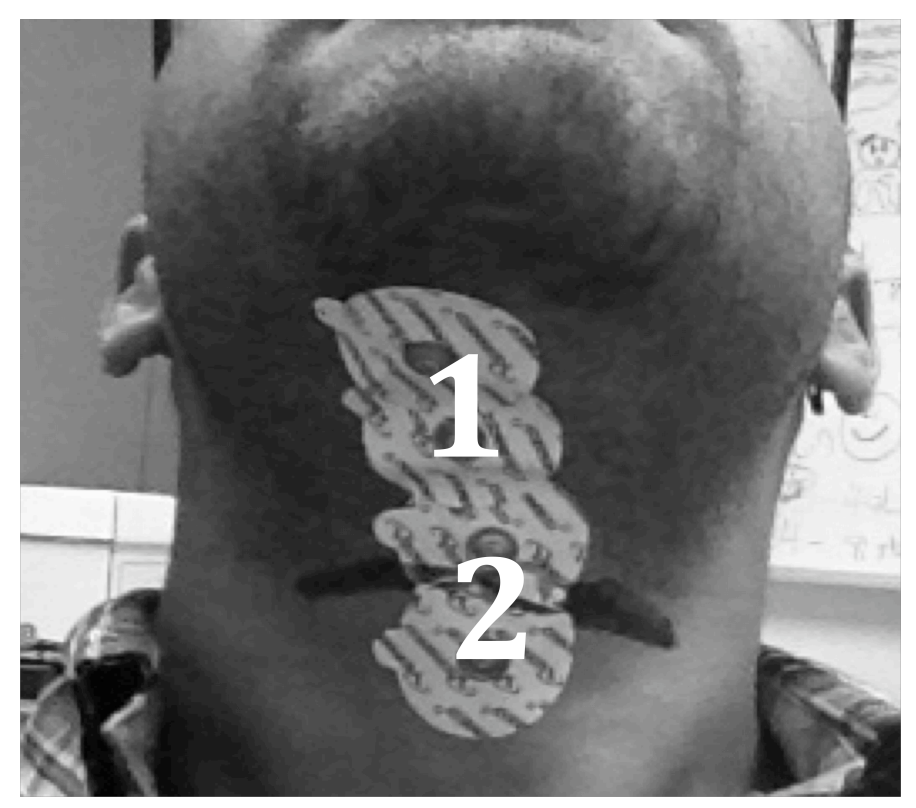

Figure 4: Photograph of electrode positions 1 and 2 on the neck of participant 1

\subsection{Instrumentation}

The EMG electrodes (Vermed@ ECG/EMG electrodes with 10\% Chloride wet gel) were positioned on the participant's neck as shown in Figure 4:

1. 1cm lateral to the submental midline (channel 1).

2. $1 \mathrm{~cm}$ lateral to the neck midline at the superior-most location prior to the start of the submental surface (channel 2).

Channel 1 was sensitive to suprahyoid strap and tongue root musculature, while channel 2 recorded existing strap musculature (possible mixture of infrahyoid and suprahyoid). Additionally, a piezo crystal respiratory effort sensor kit (Grass Technologies, Model 1370G-KIT) was placed around the lower thoracic cage to capture the participant's 
chest movement during breathing. Since the EMG signal is on the order of tens of microvolts and most of its energy occurs in the range of 10 to $500 \mathrm{~Hz}$, the EMG signals from channels 1 and 2 were amplified (gain of 10,000) and bandpass filtered (10-1000 $\mathrm{Hz}$ ) in hardware (Astro-Med Inc., Grass, P55 A.C. Preamplifier) to increase the signalto-noise ratio (SNR). The respiratory signal was amplified (gain of 1,000) and bandpass filtered $(0.1-10 \mathrm{~Hz})$. Both the EMG and respiratory signals were passed through a line filter to minimize $60 \mathrm{~Hz}$ noise. The participant also had control over a synchronization (sync) trigger that outputted a digital time stamp to mark the beginning and end of each token. This was done to ensure that the token rehearsals were not included in the data collection. The audio signal was recorded using a USB microphone (Blue Microphones, Snowball-MW) positioned about $15 \mathrm{~cm}$ from the participant. Figure 5 summarizes the experimental setup and signal acquisition. Participants were asked to hold their necks still and backs at a 90 degree angle while performing these tasks to avoid noise due to head movement.

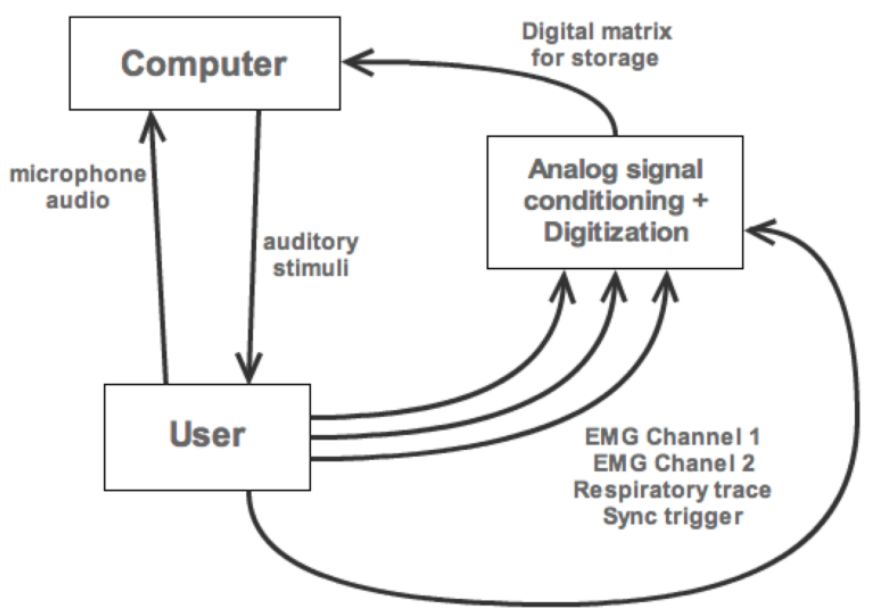

Figure 5: Block diagram depicting flow of data in experimental setup 


\subsection{Signal processing}

\subsubsection{Digitization}

The EMG and respiratory signals were all digitized at a rate of $5 \mathrm{kHz}$ via a data acquisition card (National Instruments, USB NI-6210) and the audio signal was digitized at $22050 \mathrm{~Hz}$ prior to storage on a research computer. In total, 5 channels of data were digitized by the card and stored, all time-synchronized:

1) EMG from electrodes at position 1 in Figure 4.

2) EMG from electrodes at position 2 in Figure 4.

3) Respiratory signal from the belt

4) Audio signal from the microphone

5) Sync trigger

\subsubsection{Fundamental frequency extraction}

The robust algorithm for pitch tracking (RAPT) was used to extract the full fundamental frequency (F0) contour of each audio token [39]. The RAPT parameters were adjusted to maximize the accuracy of the algorithm while accounting for the fastest variations in fundamental frequency. The duration of the frame size was set to $0.0625 \mathrm{~s}$ and the duration of the correlation window was set to $0.0625 \mathrm{~s}$ to maximize the accuracy of the F0 and VS predictions while still allowing sufficiently precise traceability of changing F0. The auditory stimuli were all in the range of $90-360 \mathrm{~Hz}$ and the voiced speech of adult males typically lies between 85 and $180 \mathrm{~Hz}$, so the maximum possible fundamental frequency was set to $380 \mathrm{~Hz}$ and the minimum possible 
fundamental frequency was set to $80 \mathrm{~Hz}$. All frequency values were converted to semitones.

\subsubsection{De-noising}

Visualizing the EMG signals during recording revealed a periodic noise pattern characteristic of ECG. ECG contamination induces significant errors with respect to the signal amplitude for low-activity signals. It was necessary to suppress this ECG signal without eliminating the low frequency components of the EMG. The frequency ranges of ECG and EMG overlap, so high-pass filtering was not a viable option. Also, a separate reference ECG recording was not available to adaptively filter out the noise, so some form of time-frequency analysis was required. The wavelet transform is a timefrequency representation of a signal that improves upon the shortcomings of the Short Time Fourier Transform (STFT). In particular, the discrete wavelet transform (DWT) can be used to remove artifacts that occupy similar frequency bands as the desired signal. We used the 'Daubechies 6' as the basic wavelet [40] with 8 decompositions. When we visually inspected the decompositions, we observed 2 dominant manifestations of ECG in the $7^{\text {th }}$ and $8^{\text {th }}$ detail. The coefficients of the $7^{\text {th }}$ and $8^{\text {th }}$ detail were therefore nullified and the signal was reconstructed with the remaining coefficients. Figure 6 shows a snippet of infrahyoid EMG taken from the speech tokens of Participant 1 before and after de-noising. This method appeared to perform well visually and, more importantly, we also found that it significantly improved the performance of our models. Figure 7 shows an example of a phrase token said by Participant 3 after the EMG was de-noised. The audio waveform in the first row corresponds to the first token from Figure 3. The second row shows the resulting F0 contour output from RAPT. The third and fourth rows show the suprahyoid and infrahyoid EMG respectively, while the final row shows the respiratory trace. 


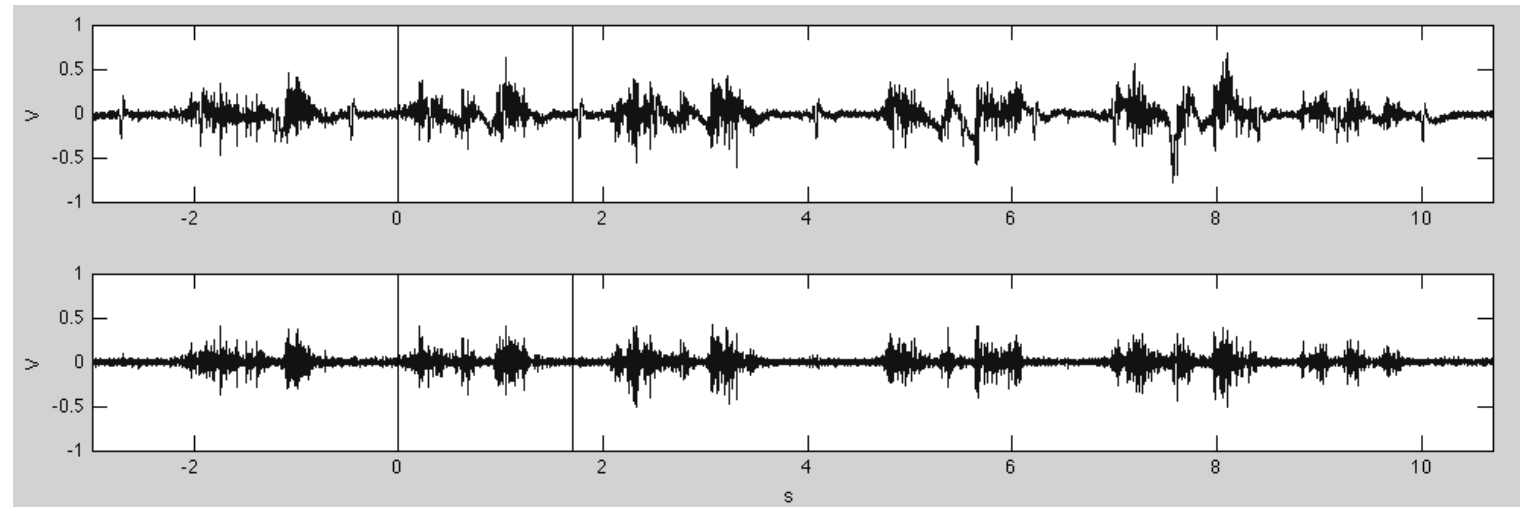

Figure 6: Infrahyoid EMG before (top) and after (bottom) wavelet denoising
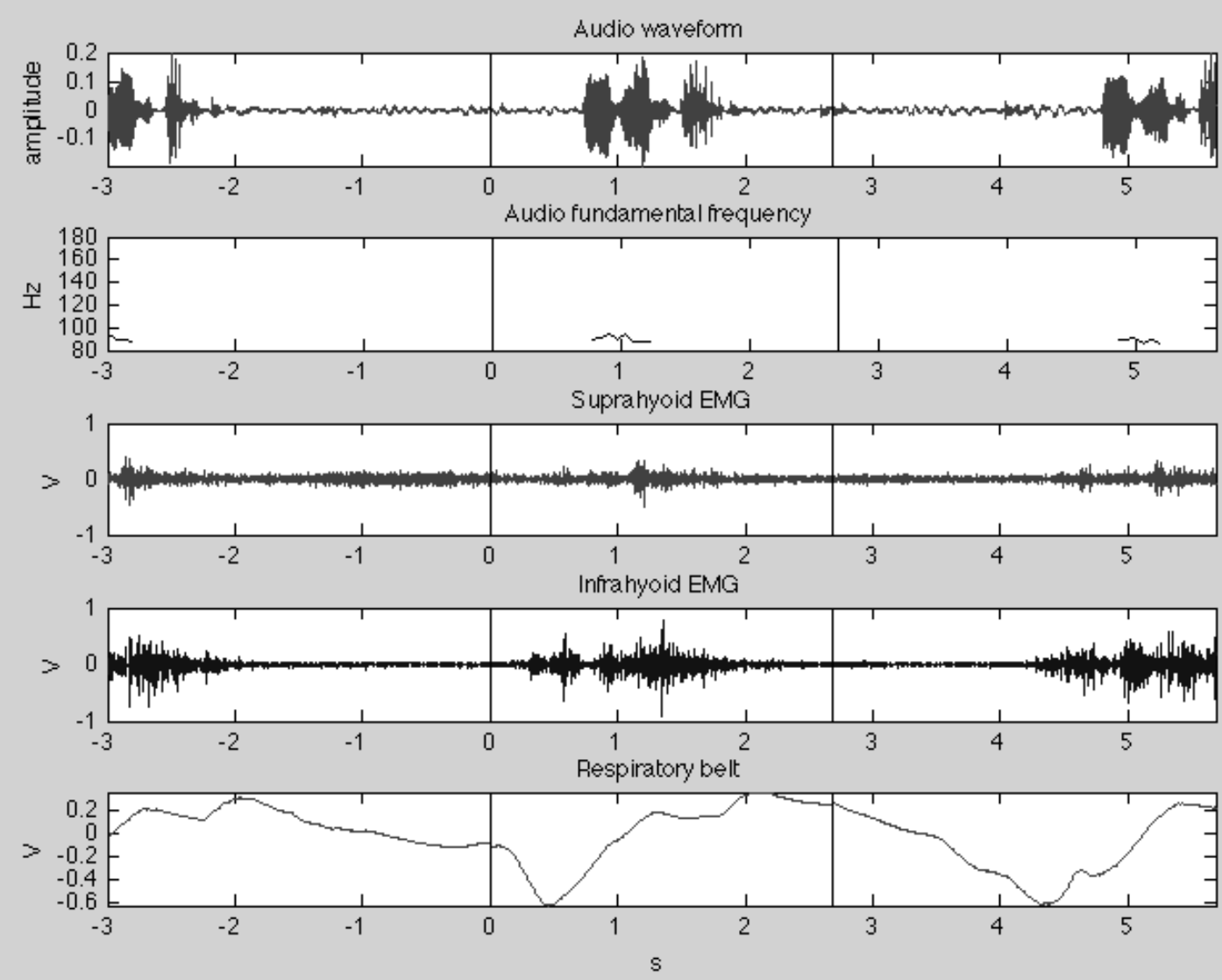

Figure 7: Sample phrase token from participant 3 


\subsection{Segmentation}

After the data collection, the acquired samples were converted into features that could be used for classification. Segmentation of each token proceeded as follows: the sync signal was used to identify the boundaries of each vocalization. Each token was selected by including one second of data from either side of the time stamps provided by the sync signal. The token signals were further segmented into $500 \mathrm{~ms}$ intervals (2560 samples in each segment) and a single feature calculated from each segment. The segment window was incremented by $125 \mathrm{~ms}$ (640 samples) for the next feature. The segment length was chosen as a trade-off between SNR and ability to follow the changing signal [41]. The inter-segment overlap was chosen in order to ensure that a control command can be generated before the clinically recognized maximum delay of 200-300 ms [42]. This segmentation approach was taken for each of the EMG channels as well as for the F0 contour, audio and respiratory belt data.

\subsection{Feature extraction}

Two feature vectors were constructed: one containing features from the 2 channels of EMG and the other containing features from the respiratory trace.

\subsubsection{EMG features}

Four kinds of features were extracted from each EMG segment to construct the first feature vector: the root mean square (RMS) value, mean amplitude value (MAV), autoregressive (AR) model coefficients and waveform length (WL). AR model coefficients were computed for a $4^{\text {th }}$ order model. The waveform length is represented mathematically as: 


$$
l=\sum_{k-1}^{N}\left|\Delta x_{k}\right|=\sum_{k=1}^{N}\left|x_{k}-x_{k-1}\right|
$$

\section{Equation 1: Waveform length}

Here, $x_{k}$ refers to the amplitude of the kth sample in the segment. WL provides a measure of the complexity of the EMG waveform in each segment. Since we used 2 channels of sEMG this provided a total 14 features per segment. These features were chosen because of their prior use in the literature to estimate muscle tension $[43,44]$.

\subsubsection{Respiratory trace features}

The respiratory trace represents the changes in thoracic diameter that occur during breathing. A positive slope indicates inhalation, while a negative slope indicates exhalation. Similarly, a flat slope indicates no change. To detect patterns related to inhalation and exhalation, five features were extracted from the respiratory trace to construct the second feature vector: 1) the maximum value of the derivative, 2) the minimum value of the derivative, 3) the maximum value of the trace, 4) the minimum value of the trace and 5) the integral of the trace.

\subsection{Label vectors}

The term label refers to the true value of a particular property that we are trying to predict. Labels were extracted from each segment of the F0 signal.

\subsubsection{Fundamental frequency}

Two labels were extracted relating to the fundamental frequency: 1) Continuous fundamental frequency (CF0): The frequency label was extracted by calculating the 
mean of the final $125 \mathrm{~ms}$ of each segment of the F0 contour. The continuous values were converted from $\mathrm{Hz}$ into semitones (st.) using the formula:

$$
s t=12 \log _{2}(b / a)
$$

\section{Equation 2: Conversion from $\mathrm{Hz}$ to semitones}

where $b$ refers to the value to be converted and $a$ refers to the value of the base tone. For all calculations in this study, $90 \mathrm{~Hz}$ was used as the base tone. 2) Discrete fundamental frequency (DF0) was also extracted: The continuous fundamental frequency was divided into discrete ranges as shown in Table 4 to generate discrete values that could be used as classes.

\section{Table 4: F0 Class divisions}

\begin{tabular}{|c|c|}
\hline Class & Range in $\mathbf{s t}$ \\
\hline 1 & -0.5 to 0.5 \\
\hline 2 & 0.5 to 1.5 \\
\hline 3 & 1.5 to 2.5 \\
\hline$\cdot$ & $\cdot$ \\
\hline$\cdot$ & $\cdot$ \\
\hline$\cdot$ & $\cdot$ \\
\hline 23 & 22.5 to 23.5 \\
\hline 24 & 23.5 to 24.5 \\
\hline
\end{tabular}

\subsubsection{Voicing state}

Two labels related to the voicing state were extracted: 
1) Voicing state (VS) was set to 0 or 1 for all voiced or unvoiced segments respectively as determined by the RAPT pitch tracker.

2) Change in voicing state (CVS) was computed as the derivative of the VS and could therefore take on 3 values as summarized in Table $\mathbf{5}$.

Table 5: CVS values and definitions

\begin{tabular}{|c|c|}
\hline CVS & \\
\hline-1 & Definition \\
\hline 0 & offset of \\
\hline 1 & onseech of \\
& speech \\
\hline
\end{tabular}

\subsection{Modeling}

The segmentation process yielded a 14-dimensional EMG feature vector and a 3dimensional respiratory trace feature vector. Four 1-dimensional label vectors were also created. Support Vector Machines (SVM) were used with the feature vectors as inputs to predict the 4 labels using the LibSVM tool [45] in the Matlab 2010a environment. The RBF kernel was used in the SVM. This was a reasonable choice since RBF performs non-linear mapping of the samples and its small number of hyper parameters results in a reduction of the complexity of the model [46,45]. The LibSVM implementation of multi-class SVM uses a one-versus-one strategy to solve a multiclass problem. 


\subsubsection{Fundamental frequency}

SVM regression and classification were used with the EMG feature vector to predict the CF0 and DF0 labels respectively. This analysis was conducted 1) for all the data together, and 2) for each modality individually to determine how much of an effect the vocalization modality had on the classification accuracy. These configurations are summarized in Figure 8.

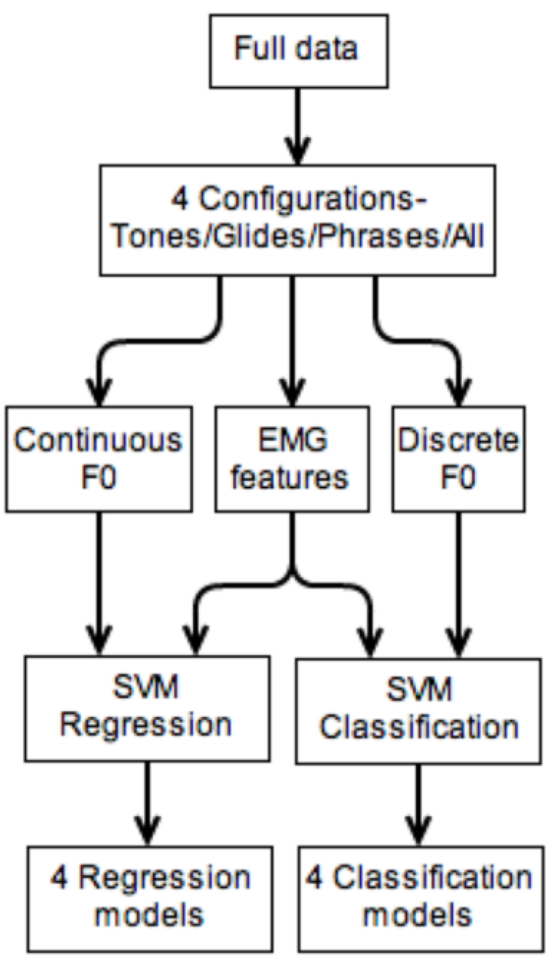

Figure 8: Data analysis configurations for predicting fundamental frequency (F0)

\subsubsection{Voicing state}

SVM classification was used to predict VS and CVS from the EMG feature vector and the respiratory trace vector individually. Only the phrases data set was used for this part of the study. 


\subsubsection{Training and testing data}

For each configuration described above, bootstrap augmentation was used to compensate for unbalanced class distributions [47]. $80 \%$ of the full feature vector and corresponding label vector rows were randomly selected and used as training data, while the remaining $20 \%$ was used as testing data. We used 8-fold cross validation with grid search on the training data to find the optimal SVM parameters $C$ and $\gamma$. A model was then generated using these parameters with the training data. This model was used to predict the labels for the testing data.

\subsubsection{User-independent training data}

We also explored the possibility of using the data from the 2 participants with the best performance as the training set for each of the other participants. The steps described in the preceding section are outlined in Figure 9. 


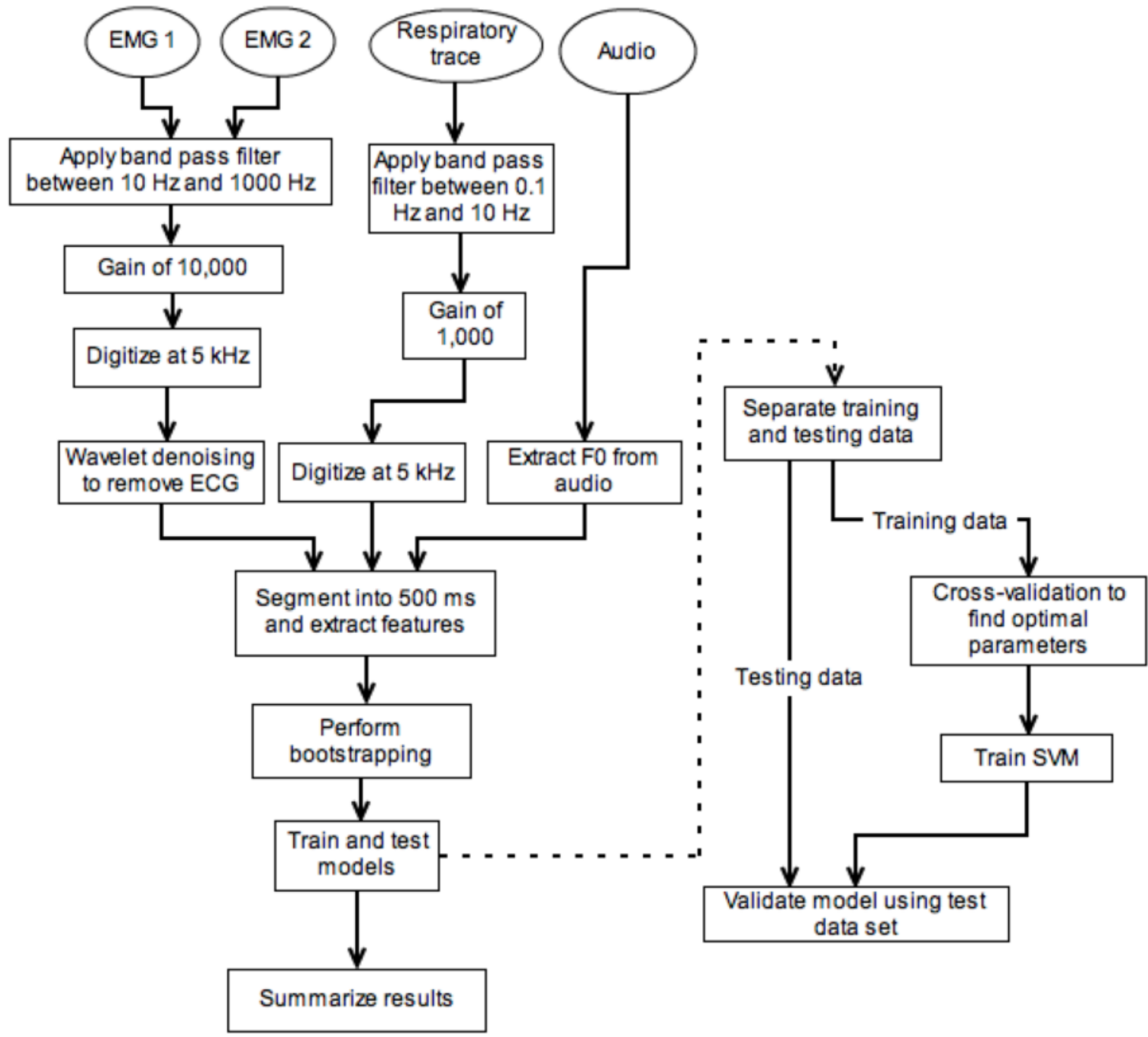

Figure 9: Process flow steps outlining the techniques describing the Methods 


\section{Chapter 3 - Results}

\subsection{Predicting fundamental frequency}

\subsubsection{Full data set}

Figure 10 shows the results of regression and classification for the prediction of F0. Average MSE of $8.21 \pm 3.5$ and $12.54 \pm 3.8$ were achieved for regression and classification, respectively, with all the participant data.

\subsubsection{Individual modalities separately}

Figure 11 shows the results of classification for the models trained on glides, tones and phrases separately. MSE of $5.92 \pm 3.6,2.93 \pm 1.1$ and $8.85 \pm 3.2$ semitones $^{2}$ were achieved for the models trained on glides, tones and phrases respectively. We achieved an average $\mathrm{R}^{2}$ of $0.93 \pm 0.03$ for the tones model and $0.78 \pm 0.04$ for the phrases model. An RMSE of $2.94 \pm 0.51$ semitones was achieved for the phrases models.

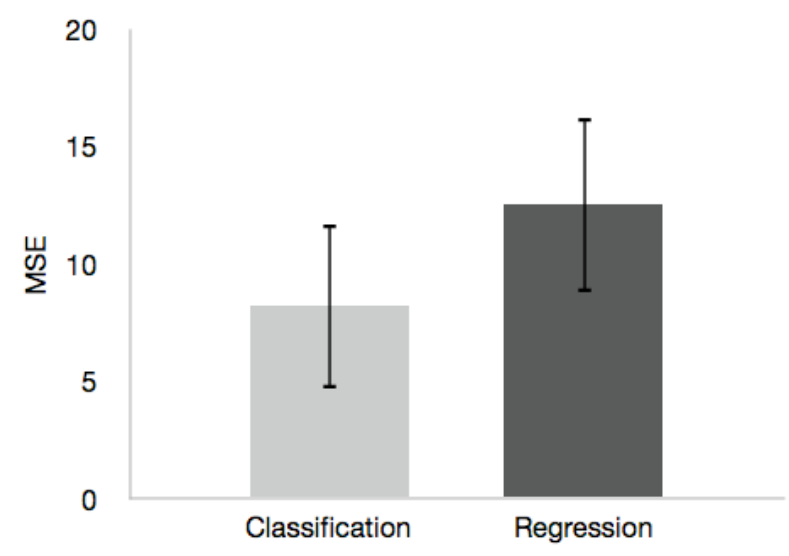

Figure 10: Average mean squared error of classification and regression models for full participant data $(\mathrm{N}=8)$ 


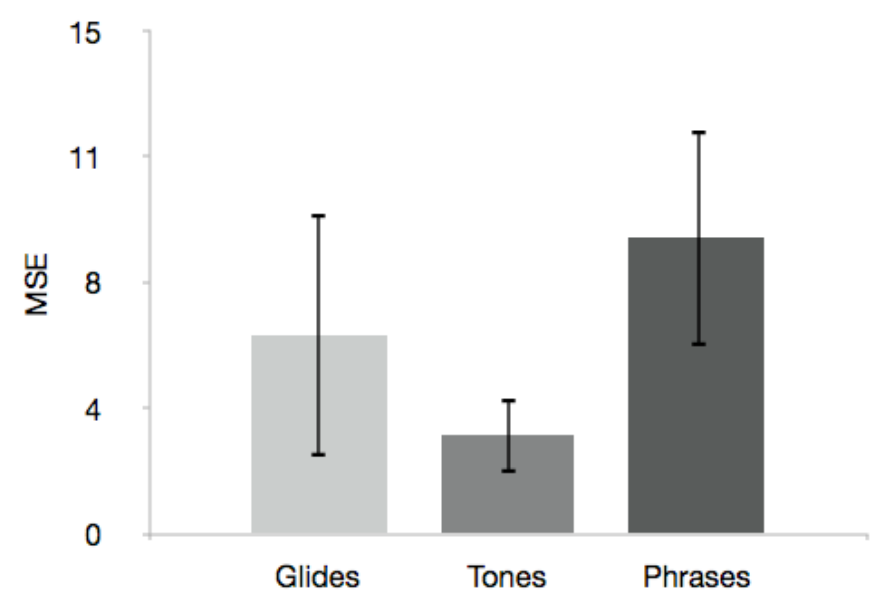

Figure 11: Average mean squared error of models trained on individual modalities $(\mathrm{N}=8)$

\subsection{Predicting voicing state}

\subsubsection{Using EMG}

An average cross-validation accuracy of $78.05 \pm 6.3 \%$ was achieved for prediction of voicing state with an average specificity of $70.5 \pm 12.1 \%$ and a sensitivity of $85.68 \pm$ $5.8 \%$. Figure 12 compares the sensitivity and specificity of the models.

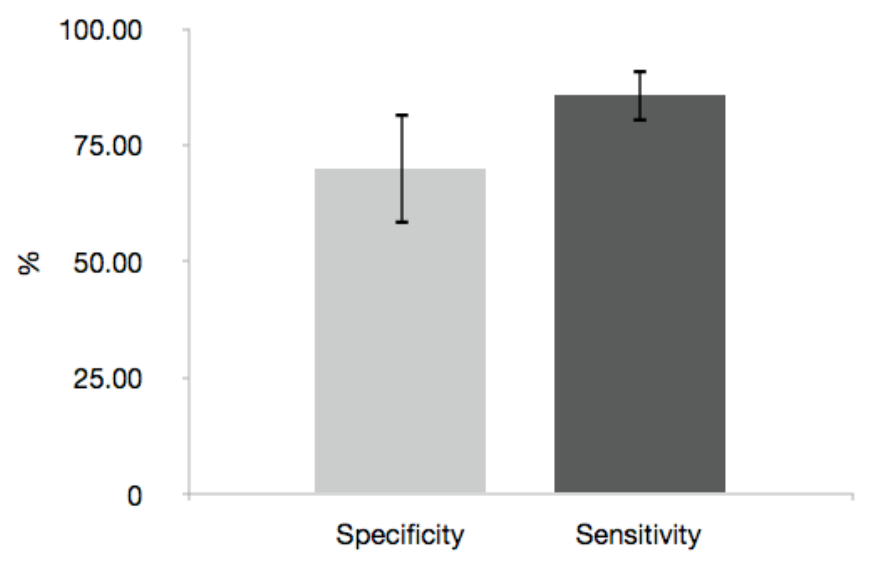

Figure 12: Specificity and sensitivity of voicing state prediction using EMG

$$
(\mathrm{N}=8)
$$




\subsubsection{Using respiratory belt}

Figure 13 shows the performance of the CVS prediction from the respiratory trace. An average cross-validation accuracy of $65.24 \pm 7.8 \%$ was achieved for CVS with average offset, no change, and onset accuracies of $80.39 \pm 9.9 \%, 44.79 \pm 5.8 \%$ and $72.79 \pm$ $13.5 \%$ respectively.

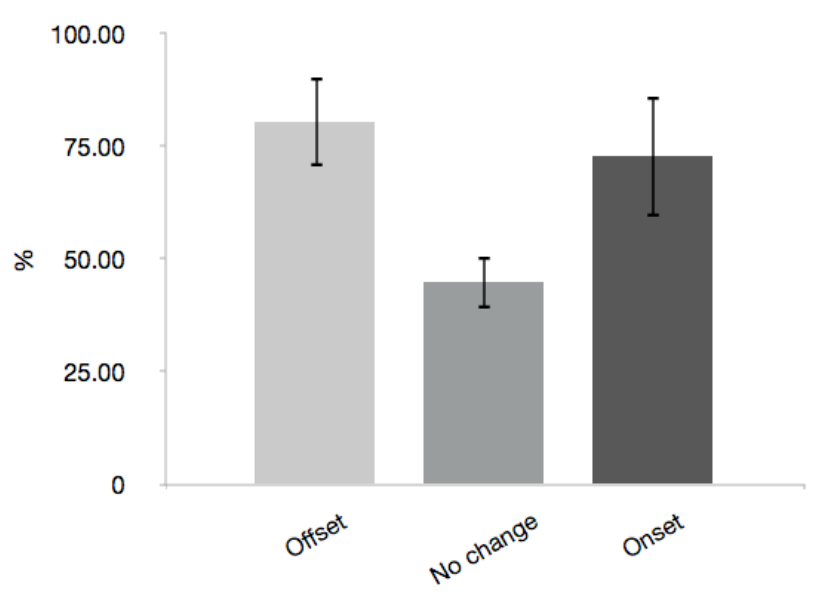

Figure 13: Performance of voicing state prediction trained on respiratory $\operatorname{trace} \operatorname{data}(\mathrm{N}=8)$

\subsection{User Independent Training Data}

We achieved an average MSE of $80.29 \pm 30.98 \%$ for F0 classification using data from the two participants with the best performance as the training set (Participants 7 and 10). Figure 14 shows how this compares with the user-specific training data. 


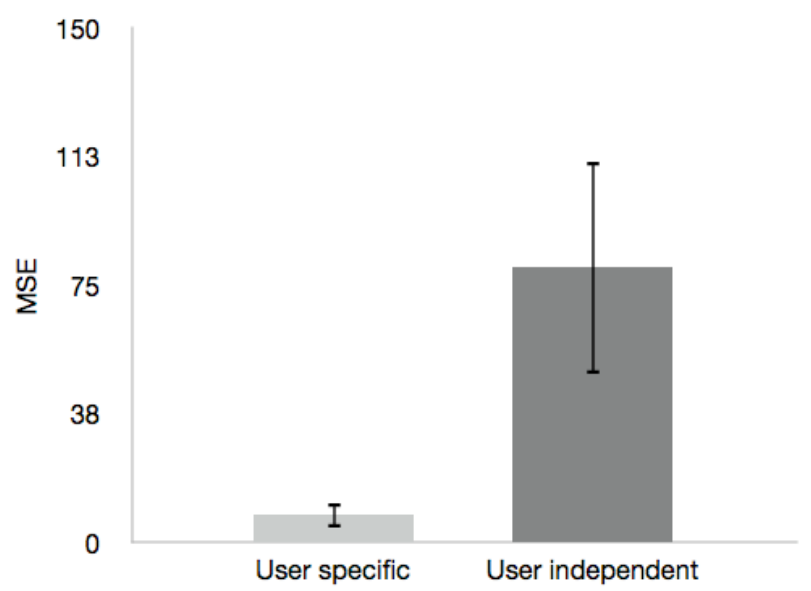

Figure 14: MSE comparison for user-specific and user-independent training $\operatorname{data}(\mathrm{N}=8)$ 


\section{Chapter 4 - Discussion}

\subsection{Predicting fundamental frequency}

\subsubsection{Regression versus classification}

Because the DF0 prediction was a multi-class classification problem, the classification accuracy does not provide a complete picture of the performance of the classifier. The MSE provides a measure of the distance by which misclassifications occur, as well as a way to compare the performance of the F0 classification with regression. On average, SVM classification predicted F0 with a MSE of $8.21 \pm 3.5$ while SVM regression performed with a MSE of $12.54 \pm 3.8$. These results show that, while the regression model offers a smoother pitch output, the classification model provides higher precision in the identification of user intention. In the future, the appropriate prediction model could be selected based on the requirements of the specific application. From this point forward, any reference to the performance of our model in predicting F0 will be about the classification model.

\subsubsection{Comparison to chance}

In order to compare the performance of our classifier to chance, a simulation was conducted as outlined in Muller-Putz et al. [48]. Two equally-sized separate random class label vectors were generated to contain normally distributed classes in the range 1 to 24 , and the MSE was calculated between them. The smallest training label vector size used in this study was 1,000 , which corresponded to a cross-validation vector size of 875, so this size was also used for the simulation. The procedure was repeated 10,000 
times yielding an MSE $>=87.39$ for $99 \%$ of the trials. The average MSE of 8.21 that we achieved in this study lies well below this threshold, indicating that the model performs better than chance.

\subsubsection{Comparison to the literature}

As described in the Background section, we only found three studies attempting to accurately predict F0 from correlated signals, physiological or otherwise [32,14,28]. Shipp et al. used logistic regression to achieve an average $\mathrm{R}^{2}$ of 0.93 using intramuscular cricothyroid (CT) EMG (the researchers used only tones in the data collection, so we can only compare the results of our tone modality to theirs). Our study paralleled the finding of Shipp et al., achieving an average $\mathrm{R}^{2}$ of $0.93 \pm 0.03$ for tones. In contrast, Saikachi et al. achieved an $\mathrm{R}^{2}$ of $0.45 \pm 0.09$ using EL RMS amplitude, while Nakamura et al. reported an average $\mathrm{R}^{2}$ of 0.13 using Gaussian Mixture Models with surface EMG of different facial muscles (both studies focused on phrases so we can compare the performance of our phrase models to their results). We achieved an average $\mathrm{R}^{2}$ of $0.78 \pm$ 0.04 for the phrase modality, suggesting that our decision to explore pattern recognition with neck muscle EMG has merit.

\subsubsection{Standalone evaluation}

The literature indicates that only pitch differences of more than three semitones can be discriminated reliably in speech [49]. This suggests that misclassifications by less than three semitones would not affect the intended meaning of an intonational change during speech. The average RMSE of our classification model is less than three, which indicates that the model performs quite promisingly offline. It is expected that this RMSE would be a bit larger for patients since the amount of infrahyoid and suprahyoid musculature available post-laryngectomy is very case-specific. 


\subsection{Sources of error for F0 prediction}

\subsubsection{Unbalanced class distribution}

This study required participants to vocally reproduce auditory stimuli that were difficult to match precisely and often sounded very similar to each other. Musical training was not a prerequisite for inclusion in the study so we made several considerations in the data collection phase to make the stimuli as reproducible and distinguishable as possible. We used 2 major scales to present the 12 notes on each octave rather than the chromatic scale. We presented glides first to acclimatize participants to the pitch recognition and reproduction tasks required of them in the study. We also used whole tone distances for the tone modality rather than semitone distances. In spite of these considerations, there was significant variation in the performance of participants. Participants tended to perform satisfactorily near the middle of the 90-360 Hz range, but they struggled to attain and maintain frequencies towards the low and high ends. As a result, there was significantly more data available for the mid- range than for the low and high ends. Additionally, even though the sentences were chosen to include various stress placements, phrases cannot possibly provide a comprehensive coverage of all the possible $\mathrm{F} 0$ in the targeted range. For this reason, the problem of class imbalance affects the phrase models to an even greater degree than the tone and glide models. Even though bootstrap augmentation was used to compensate for the imbalance, the results obtained for accuracy and MSE for participants who had better pitch perception and who could maintain a specific F0 level with little variation were much better than the rest. In retrospect, real-time visual feedback of participant F0 could have been useful for the tones part of the study. We speculate that the significantly superior performance of the classification model is at 
least partly attributable to the use of bootstrap augmentation to supplement the more poorly represented classes. In the future, real-time evaluation of this study could provide more insight into the potential of the proposed compensation strategy.

\subsubsection{Use of sEMG rather than intramuscular EMG}

The extrinsic laryngeal muscles we targeted are much less correlated with F0 than the intrinsic muscles, which Shipp et al. [28] found were the single most important physiologic correlates of vocal frequency. To add to this, surface EMG is less sensitive than intramuscular EMG since it picks up signals from different motor units as well as physiologic noise as it travels through different tissues [50]. We chose to avoid intramuscular EMG because of its invasiveness. In spite of this, our results show that we were able to achieve results comparable to Shipp et al. [28], a finding that supports future non-invasive exploitation of surface EMG for pitch modulation.

\subsubsection{Vocalization modality}

An important feature of this study is that we investigated the performance of models trained on different modalities of vocalization. Previous analyses of the role of laryngeal muscles in F0 control have used either tones [28], glides [16] or phrases [18]. Because of differences in experimental methods, these studies all report different findings about the role of laryngeal muscles in F0 control. Strap muscle activity is related to the dynamic aspects of the vocalization process so any observations are dependent on the modality of the vocal production studied. Any gesture of speech or non-speech that requires a change in the vertical position or fixation of the hyoid bone tends to show a change in the activity of the attached muscles. For example, jaw opening and closing, tongue lowering, raising, protrusion and retraction all involve either activation or inhibition of the sternohyoid [51]. It is clear from our study that the performance of the models 
trained on tones alone is significantly greater than that of the models trained on either glides or phrases. We can partly attribute the lower performance of the models trained on the entire data set to noise in the EMG due to jaw and tongue movements inherent in glides and speech. Nonetheless, the models trained on phrases still predict pitch with an RMSE of 2.94 semitones and are therefore still useful for implementation in an electrolarynx.

\subsubsection{Pitch tracker error}

Pitch trackers are prone to errors like pitch halving and pitch doubling, as well as significant noise due to voicing decision errors. The RAPT pitch tracker algorithm was used to compute all our labels, so these types of errors can negatively affect the performance of all our models.

\subsubsection{Electrode preparation}

The electrode contact impedance for male participants with beards was greater than that for those without. In addition the geometry of the neck area accommodated our large EMG electrode pads better for some participants than for others. This may have contributed to some of the variation in user-specific model performance.

\subsection{Predicting voicing state}

\subsubsection{Using EMG}

We attempted to predict voicing state in two ways. The higher classification accuracies of $78.05 \pm 6.3$ were achieved using features from EMG. The chance threshold for a 2class experiment with our smallest sample size of 6000 is about 50.06 as calculated from 
Muller-Putz et al. [48], so our classifier does perform significantly better than random. Nakamura et al. [32] achieved an average classification accuracy of 79.8 using facial muscle EMG, so our results are not significantly different. A significant observation is the fact that, as Figure 7 shows, EMG activity precedes vocalization by as much as $400 \mathrm{~ms}$ and persists after the end of vocalization. This phenomenon may have contributed to the low specificity of the model. We expected, however, to achieve a higher sensitivity since EMG activity is observable at all times when there is vocalization. It should be noted, however, that we did not analyze any periods of silent rest without vocalization. All the silent periods we analyzed occurred either just before or just after a vocalization. This meant that most of the frames in our analysis contained vocalization-related EMG activity, resulting in a reduction in the separability of voiced and unvoiced classes. Including more silent periods without any vocalization in the training data could well improve the sensitivity and overall performance of our model. We also speculate that our results could be significantly improved by including features from the facial muscle electrode positions used by Nakamura et al. [32].

\subsubsection{Using respiratory trace}

The chance threshold for a 3 -class problem with a sample size of 1000 is $31.2 \%$ using the simulation described in Muller-Putz et al. [48], confirming that our results exceed chance. Nonetheless, our results are not very promising. We expected to be able to detect the inhalation that takes place at the onset of vocalization with the "onset" class. For each phrase, taking the derivative of the voicing state label yielded a single 500ms segment labeled "onset" that overlapped with the first $125 \mathrm{~ms}$ after onset of voicing. The inhalation can clearly be seen in Figure 7 and we expected that the features we chose could be able to distinguish this segment from a segment occurring during voicing. However, the overlap that subsequent voiced or "no change" segments share with this 
"onset" segment might have diminished class separability. This same issue of overlap occurs for the offset class as well. A solution to this might be to eliminate the overlap for voicing state prediction. A new feature would not be calculated every 125ms, but rather every 500ms. Since this time difference might be too large for real-time applications, we could also consider reducing the segment width to $250 \mathrm{~ms}$ or less. We did not, however, investigate the potential of these considerations to improve classification accuracy in this study.

As shown in Figure 13, the classifier performs the worst at correctly identifying segments where no change in voicing state occurs. These were very frequently misclassified.

\subsection{User independent training data}

Unfortunately, user specific data cannot be collected from non-vocal patients by the methods described in this study. For these methods to be applicable to the non-vocal population, the use of user-independent training data is a necessity. The F0 prediction for the user-independent model does perform above the chance threshold of 87.39 , but not by much. Quite likely, the poor performance is a result of the significant intersubject variability in the EMG-F0 relationship reported in the literature [30,28]. This high variability is largely due to inherent physiological differences (e.g. in muscle geometry, ratios of slow- to fast-twitch fibers, recruitment patterns and firing frequencies). As a result, the odds of misclassification for an unseen subject are greatly increased. These factors help explain the poor results that we present here. A solution to this issue might be to train the models on users pre-laryngectomy. 


\section{Chapter 5 - Future work}

\subsection{Robustness}

One area we did not investigate in this study was the incidence of noise from swallowing, yawning and other such potential false triggers. A future study might consider frequency and time-frequency domain features of EMG to distinguish swallows and yawns from vocal-related activity. Another area for further research is a methodology to remove noise due to lip, mouth and tongue movements from the EMG data. This might require the use of additional EMG channels from the face.

\subsection{User training for user-independent models}

Humans can develop skill by self-initiated error detection and correction through intrinsic and augmented feedback [29]. Able-bodied participants learned to control the infrahyoid muscles even prior to formal training [31], so it is reasonable to imagine that finer control can be developed with training. Participants in the study conducted by Goldstein et al. [5] were able to elevate strap muscle activity to increase pitch. This is contrary to the role of the infrahyoid muscles in normal speech and is evidence of a new pitch regulation mechanism being assimilated. A comprehensive pitch regulation mechanism has not yet been presented in the literature as an alternative to normal laryngeal function. However, instrumental music has been found to mirror the rhythmic and melodic patterns found in speech [52] so it is conceivable that traditional methods of musical instruction could be applied to training in speech prosody. The performance of the user-independent models could be significantly improved with user training. 


\subsection{Removal of ECG artifact}

We did not investigate the performance of the various ECG-removal algorithms presented in the literature $[53,54]$. There might be a more effective way of removing the ECG artifact than what we presented. This could have a positive effect on the performance of our models. A future study could compare the performance of competing algorithms to determine the most appropriate approach of ECG removal from neck muscle EMG. 


\section{Chapter 6 - Conclusions}

We presented the estimation of F0 and voicing state from neck muscle EMG in a machine learning paradigm. We proposed an SVM-based classification model to predict F0 using an input feature vector of 14 dimensions. Our results for prediction of F0 compare favorably with the literature, with the advantage of non-invasiveness over Shipp et al. [28] and superior performance over both Saikachi et al. [14] and Nakamura et al. [32]. We also proposed two SVM-based classification models to predict voicing state from EMG and respiratory trace, respectively. The prediction from EMG performed significantly better than that from the respiratory trace and equaled the best results reported in the literature [32]. These findings serve as useful ground work for future developments in automatic pitch modulation for electrolarynges and silent speech interfaces. 


\section{Contributions}

The scientific contributions of this thesis are as follows:

1) Creation of classification and regression models to relate laryngeal muscle activity and respiration to vocal frequency and voicing state.

2) Demonstration that EMG signals from the infrahyoid and suprahyoid muscles in adult males can be used to algorithmically predict fundamental frequency with a mean squared error of approximately 8 semitones $^{2}$ across typical male vocalization frequencies.

3) Demonstration that in adult males, voicing state can be predicted with higher accuracy from surface EMG than from respiratory trace. 


\section{References}

[1] Madden, B., Nolan, M., Burke, T., Condron, J., Coyle, E., 2010. Intelligibility of Electrolarynx Speech Using a Novel Actuator, Signals and Systems Conference, Cork, Ireland.

[2] Gray, S., Konrad, H.R., 1976. Laryngectomy: Postsurgical rehabilitation of communication. Archives of Physical Medicine and Rehabilitation 57, 140-142.

[3] Hillman, R.E., Walsh, M.J., Wolf, G.T., Fisher, S.G., Hong, W.K., 1998. Functional outcomes following treatment for advanced laryngeal cancer. Part 1 - Voice preservation in advanced laryngeal cancer. Part II - Laryngectomy rehabilitation: The state of the art in the VA System. Annals of Otology, Rhinology and Laryngology Supplement 172, 127 .

[4] Mendenhall, W.M., Morris, C.G., Stringer, S.P., Amdur, R.J., Hinerman, R.W., Villaret, D.B., 2002. Voice rehabilitation after total laryngectomy and postoperative radiation therapy. J. Clin. Oncol. 20, 2500-2505.

[5] Goldstein, E.A., Stepp, C.E., Hillman, R.E., 2007. Training effects on speech production using a hands-free electromyographically controlled electrolarynx. J. Speech Lang. Hear. Res. 50, 335-351.

[6] Watson, J.W., Schlauch, R.S., 2009. Fundamental frequency variation with an electrolarynx improves speech understanding: a case study. Am. J. Speech-Lang. Pat. $18,162-167$.

[7] Meltzner, G.S., Hillman, R.E., 2005. Impact of aberrant acoustic properties on the perception of sound quality in electrolarynx speech. J. Speech Lang. Hear. Res. 48, 766779 .

[8] Barney, H., Haworth, E., Dunk, H., 1959. An experimental transistorized artificial larynx. Bell Syst. Tech. J. 38, 1337-1356.

[9] Qi, Y.Y., Weinberg, B., 1991. Low-frequency energy deficit in electrolaryngeal speech. J. Speech Lang. Hear. Res. 34, 1250-1256. 
[10] Cole, D., Sridharan, S., Moody, M., Geva, S., 1997. Application of noise reduction techniques for alaryngeal speech enhancement, IEEE TENCON '97.

[11] Houston, K.M., Hillman, R.E., Kobler, J.B., Meltzner, G.S., 1999. Development of sound source components for new electrolarynx speech prosthesis, IEEE Proc. International Conference on Acoustics, Speech and Signal Processing, pp. 2347-2350.

[12] Ma, K., Demirel, P., Espy-Wilson, C., MacAuslan, J., 1999. Improvement of electrolarynx speech by introducing normal excitation information, Proceedings of the European Conference on Speech Communication and Technology, Budapest, pp. 323326.

[13] Goldstein, E.A., Heaton, J.T., Kobler, J.B., Stanley, G.B., Hillman, R.E., 2004. Design and implementation of a hands-free electrolarynx device controlled by neck strap muscle electromyographic activity. IEEE Trans. Biomed. Eng. 51, 325-332.

[14] Saikachi, Y., Stevens, K.N., Hillman, R.E., 2009. Development and perceptual evaluation of amplitude-based F0 control in electrolarynx speech. J. Speech Lang. Hear. Res. 52, 1360-1369.

[15] Uemi, N., Ifukube, T., Takahashi, M., Matsushima, J., 1994. Design of a new electrolarynx having a pitch control function, IEEE Workshop on Robot and Human Communication.

[16] Roubeau, B., Chevrie-Muller, C., Guily, J.L.S., 1997. Electromyographic activity of strap and cricothyroid muscles in pitch change. Acta Otolaryngologica 117, 459-464.

[17] VandenBerg, J.W., 1960. Vocal Ligaments vs Registers. Current Problems in Phoniatrics and Logopedics 1, 19-34.

[18] Atkinson, J.E., 1978. Correlation analysis of the physiological factors controlling fundamental voice frequency. J. Acous. Soc. Am. 63, 211-222.

[19] Stepp, C.E., Heaton, J.T., Rolland, R.G., Hillman, R.E., 2009. Neck and face surface electromyography for prosthetic voice control after total laryngectomy. IEEE Trans. Neural. Syst. Rehabil. Eng. 17, 146-155. 
[20] Jacobsen, S.C., Knutti, D.F., Johnson, R.T., Sears, H.H., 1982. Development of the Utah artificial arm. IEEE Trans. Biomed. Eng. 29, 249-269.

[21] Koike, Y., Kawato, M., 1995. Estimation of dynamic joint torques and trajectory formation from surface electromyography signals using a neural network model. Biol. Cybern. 73, 291-300.

[22] Saridis, G.N., Gootee, T.P., 1982. EMG pattern analysis and classification for a prosthetic arm. IEEE Trans. Biomed. Eng. 29, 403-412.

[23] Yamada, M., Niwa, N., Uchiyama, A., 1983. Evaluation of a multifunctional hand prosthesis system using EMG controlled animation. IEEE Trans. Biomed. Eng. 30, 759763.

[24] Junker, A., 1995. Brain-body actuated system, United States.

[25] Scargle, S., 1998. EMG/EEG head-computer-interface system for computer cursor control. International University of Florida.

[26] Perry, J., Bekey, G., 1981. EMG-Force relationships in skeletal mscle. CRC Critical Reviews in Biomedical Engineering 7, 1-22.

[27] Metral, S., Cassar, G., 1981. Relationship between force and integrated EMG activity during voluntary isometric anisotonic contraction. Eur. J. Appl. Physiol. 46, 185-198.

[28] Shipp, T., Doherty, T., Morissey, P., 1979. Predicting vocal frequency from selected physiological measures. J. Acous. Soc. Am. 66, 678-684.

[29] Zelaznik, H., 1996. Advances in Motor Learning and Control. Human Kinetics.

[30] Heaton, J.T., Goldstein, E.A., Kobler, J.B., eitels, S.M., Randolph, G.W., Walsh, M.J., Gooey, J.E., Hillman, R.E., 2004. Surface electromyography activity in total laryngectomy patients following laryngeal nerve transfer to neck strap muscles. Ann. Otol. Rhinol. Laryngol. 113, 754-764. 
[31] Kubert, H.L., Stepp, C.E., Zeitels, S.M., Gooey, J.E., Walsh, M.J., Prakash, S.R., Hillman, R.E., Heaton, J.T., 2009. Electromyographic control of a hands-free electrolarynx using infrahyoid muscles. J. Commun. Disord. 42, 211-225.

[32] Nakamura, K., Janke, M., Wand, M., Schultz, T., 2011. Estimation of fundamental frequency from surface electromyographic data: EMG-to-F0, IEEE International Conference on Acoustics, Speech and Signal Processing (ICASSP), Prague, Czech Republic, pp. 573-576.

[33] Nolan, F., 2003. Intonational equivalence: an experimental evaluation of pitch scales, Proceedings of the 15th International Congress of Phonetic Sciences, Barcelona, pp. 771-774.

[34] Nooteboom, S., 1997. The prosody of speech: melody and rhythym, Research Institute for Language and Speech. Utrecht University, Utrecht.

[35] Castellini, C., van der Smagt, P., 2009. Surface EMG in advanced hand prosthetics. Biol. Cybern. 100, 35-47.

[36] Park, W., Kwon, S., Kim, J., 2010. Real-time estimation of thumb-tip forces using surface electromyogram for a novel human-machine interface, IEEE International Conference on Robotics and Automation, pp. 205-210.

[37] Yang, D., Zhao, J., Gu, Y., Jiang, L., Liu, H., 2009. EMG pattern recognition and grasping force estimation: improvement to the myocontrol of multi-DOF prosthetic hands, IEEE/RSJ International Conference on Intelligent Robots and Systems.

[38] Zhao, J., Xie, Z., Jiang, L., Cai, H., Hirzinger, G., 2005. Levenberg-Marquardt Based Neural Network Control for a Five-fingered Prosthetic Hand, International Conference on Robotics and Automation, pp. 4482-4487.

[39] Talkin, D., 1995. A robust algorithm for pitch tracking (RAPT), in: Kleijn, W.B., Paliwal, K.K. (Eds.), Speech coding and synthesis. Elsevier Science, Amsterdam, pp. 495-518.

[40] Daubechies, I., 1992. Ten lectures of wavelets. SIAM. 
[41] St-Amant, Y., Rancourt, D., Clancy, E.A., 1996. Effect of smoothing window length on RMS EMG amplitude estimates, Bioengineering Conference, 1996., Proceedings of the 1996 IEEE Twenty-Second Annual Northeast, pp. 93-94.

[42] Scott, R.N., 1984. An introduction to myoelectric prostheses. UNB Monographs on Myoelectric Prostheses.

[43] Hudgins, B., Parker, P., 1993. A new strategy for multifunction myoelectric control. IEEE Trans. Biomed. Eng. 40, 82-94.

[44] Khokhar, Z.O., Xiao, Z.G., Menon, C., 2010. Surface EMG pattern recognition for real-time control of a wrist exoskeleton. Biomed. Eng. Online 9.

[45] Hsu, C.W., Chang, C.C., Lin, C.J., 2003. A practical guide to support vector classification, Technical Report. Department of Computer Science and Information Engineering, National Taiwan University, Taipei, Taiwan.

[46] Hastie, T., Rosset, S., Tibshirani, R., Zhu, J., 2004. The entire regularization path for the support vector machine. J. Mach. Learn. Res. 5, 1391-1415.

[47] Chen, J.J., Tsai, C.A., Young, J.F., Kodell, R.L., 2005. Classification ensembles for unbalanced class sizes in predictive toxicology. SAR QSAR Environ. Res. 16, 517-529.

[48] Muller-Putz, G.R., Scherer, R., Brunner, C., Leeb, R., Pfurtscheller, G., 2008. Better than random? A closer look on BCI results. International Journal of Bioelectromagnetism 10, 52-55.

[49] 't Hart, J., 1980. Differential sensitivity to pitch distance, particularly in speech. J. Acous. Soc. Am. 69, 1981.

[50] Reaz, M.B.I., Hussain, M.S., Mohd-Yasin, F., 2006. Techniques of EMG signal analysis: detection, processing, classification and applications. Biol. Proceed. Online 8, 11-35.

[51] Ohala, J., Hirose, H., 1969. The function of the sternohyoid muscle in speech, Reports of the Autumn 1969 meeting of the Acoustical Society of Japan, Tokyo, Japan, pp. 359-360. 
[52] Patel, A.D., Iversen, J.R., Rosenberg, J.C., 2006. Comparing the rhythm and melody of speech and music: The case of British English and French. J. Acous. Soc. Am. 119, 3034-3047.

[53] Taelman, J., VanHuffel, S., Spapen, A., 2007. Wavelet-independent component analysis to remove electrocardiography contamination in surface electromyography, Proceedings of the 29th Annual International Conference of the IEEE EMBS, Lyon, France, pp. 682-685.

[54] von Tscharner, V., Eskofier, B., Federolf, P., 2011. Removal of the electrocardiogram signal from surface EMG recordings using non-linearly scaled wavelets. J. Electromyogr. Kines. 21, 683-688. 